$1 \cdot 1$

\author{
أثر الأزمة المالية والاقتصادية العالمية على التجارة الخارجية

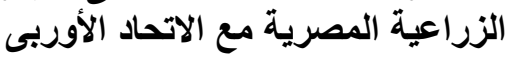 \\ رانيا عبد الفتاح الشاعر \\ معهد بحوث الاقتصاد الزراعى - مركز البحوث الزراعية الفئ
}

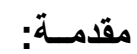

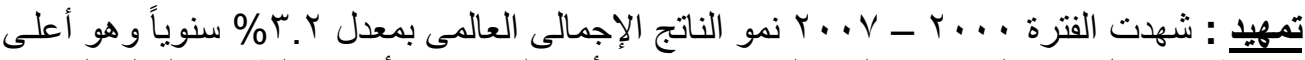

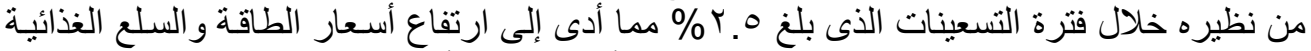

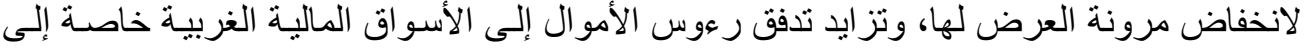

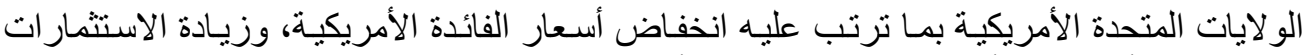

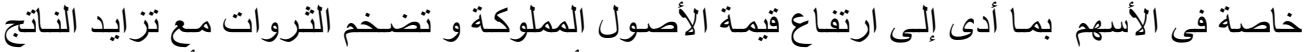

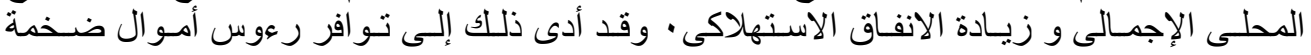

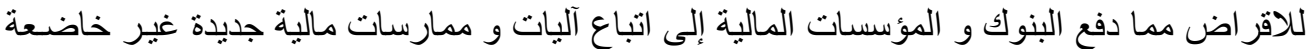

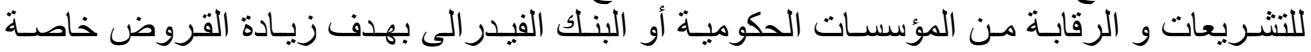

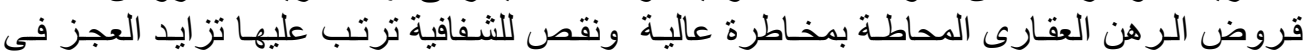

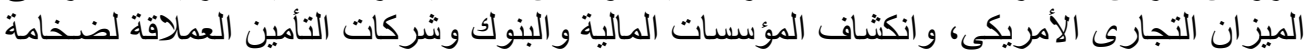

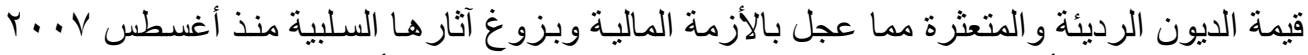

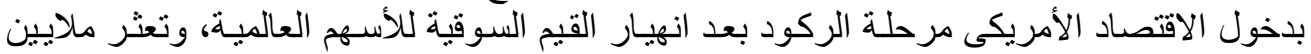

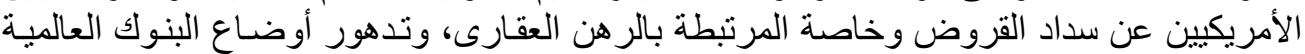

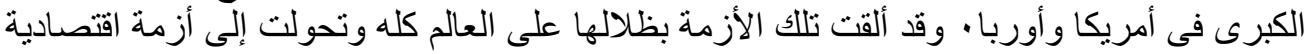

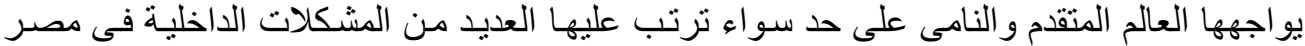

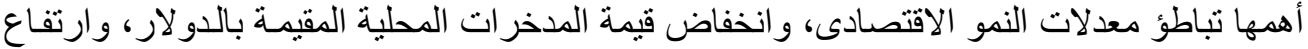

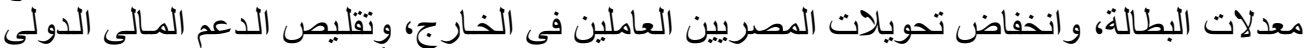

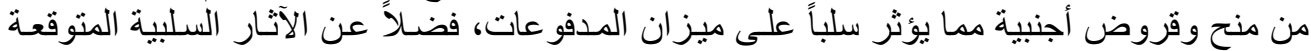

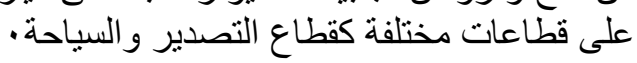

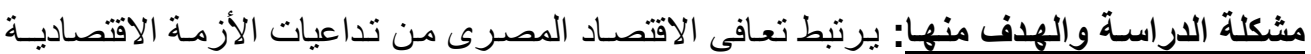

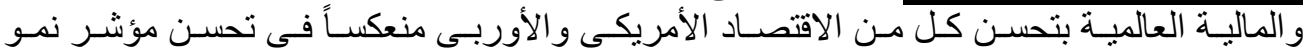

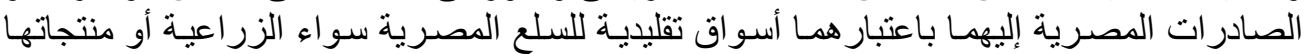

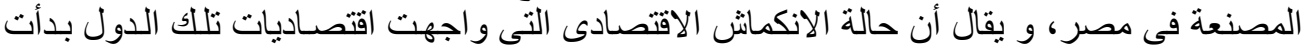

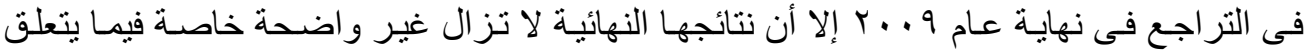

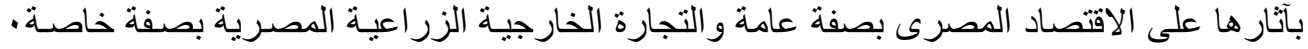

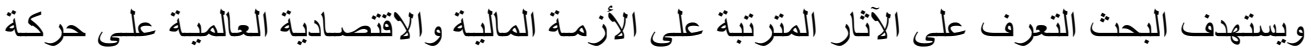

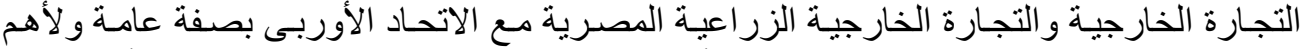

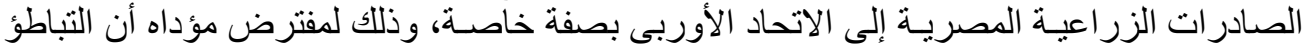

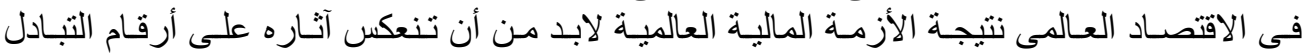

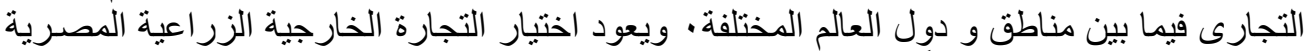

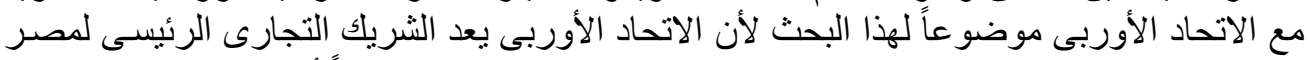

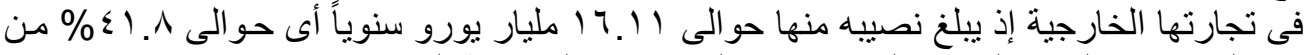

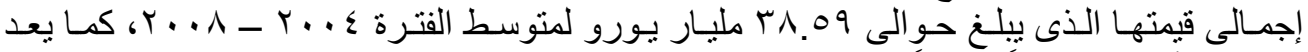

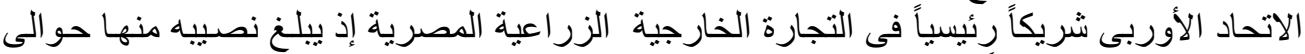

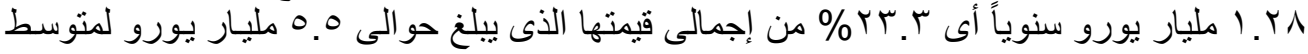

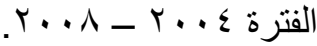

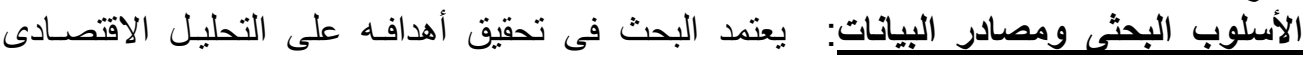

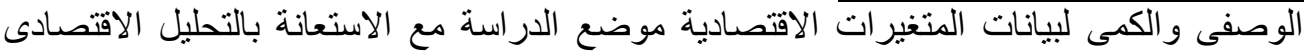

Fayoum J. Agric. Res. \& Dev., Vol.23, No.2, (B) July, 2009 
$1 \cdot 9$

الإحصائى من خلال مختلف أساليب عرض البيانات الجدوليـة والمتوسطات الحسابيـة والنسب

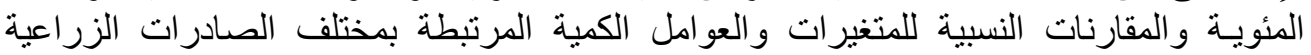
المصرية المتجهة إلى الاتحاد الأوربى. وتجدر الإشارة إلى أهم مؤشرات وات ومعايير قياس أهمية

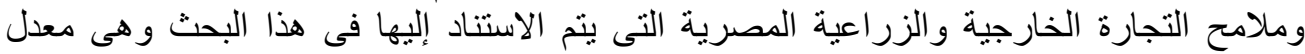

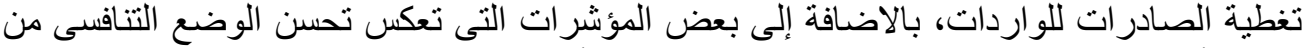
عدمه لأهم الصادر ات الزر اعية المصرية في الاتحاد الأوربى منمثلة فى :

(1) Changes in Global Market Share of Major Exports :

$\mathrm{CD}_{\mathrm{j}}=\left(\mathrm{M}_{\mathrm{it}}-\mathrm{M}_{\mathrm{i} 0}\right) * \mathrm{I}_{\mathrm{gt}}$

حيث

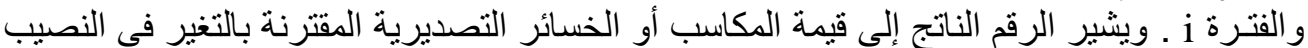
السوقى من فترة لأخرى والتى تعكس تحسن المركز التنافسى للدولة المعنية فى تصدير سلعة معينة

(2) Export Specialization Index: إلى سوق معين من عدمها. $\mathrm{ES}=\left(\mathrm{xij} / \mathrm{X}_{\mathrm{it}}\right) /\left(\mathrm{m}_{\mathrm{kj}} / \mathrm{M}_{\mathrm{kt}}\right)$

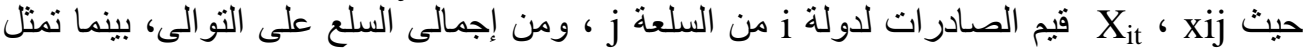

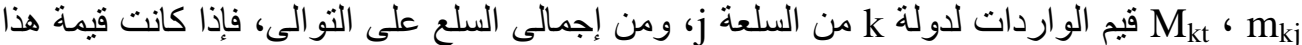

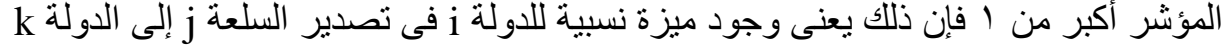

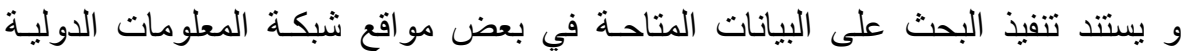

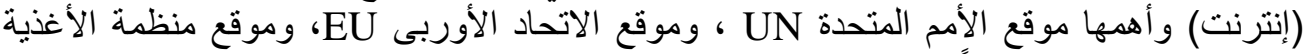

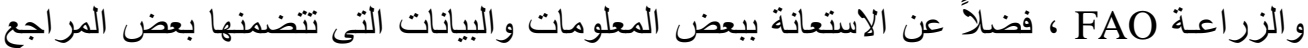

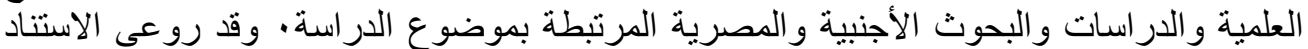

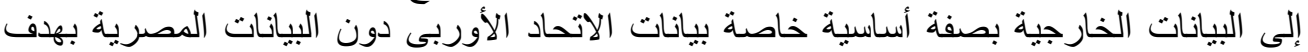

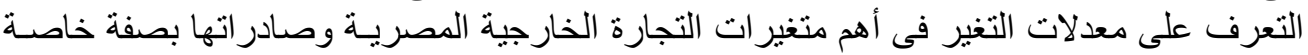

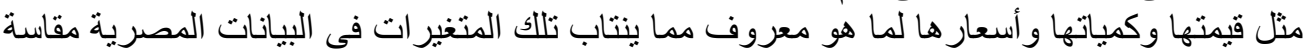

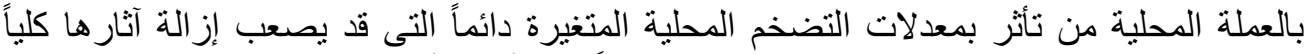
حتى فى حالة تحويلها إلى قيم حقيقية غير نقدية اعتماداً على أحد الأرقام القياسية المناسبة لذللك.

التجارة الخارجية الكلية والزراعية المصرية:

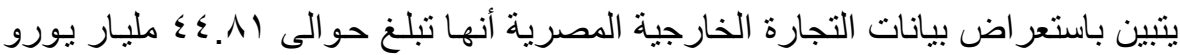

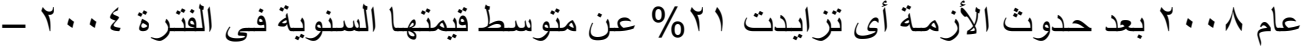

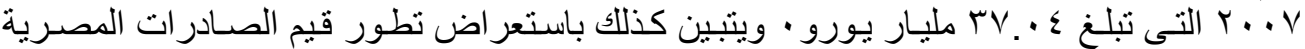

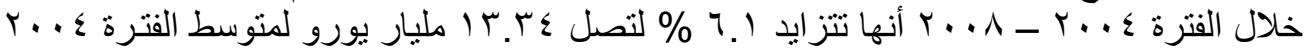

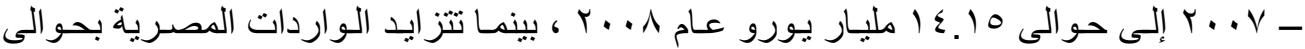

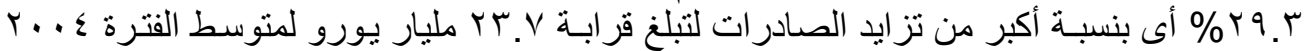

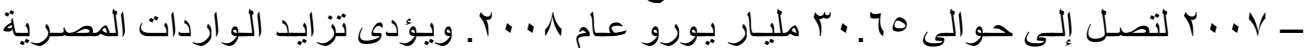

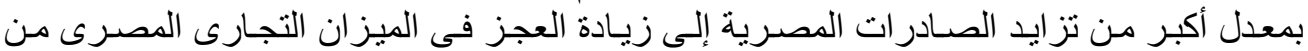

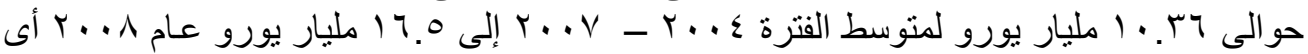

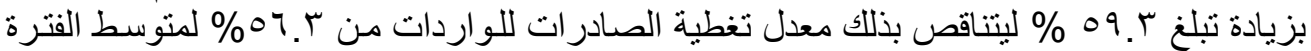

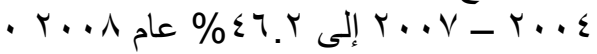

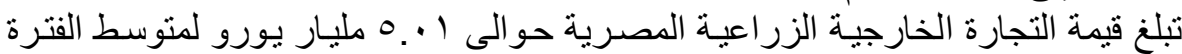

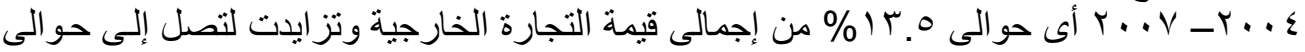

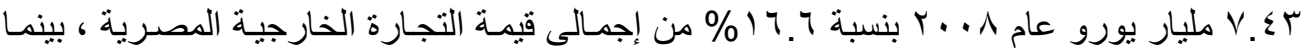

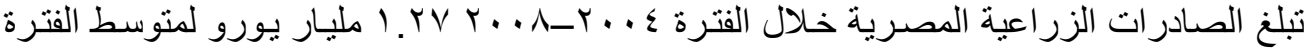

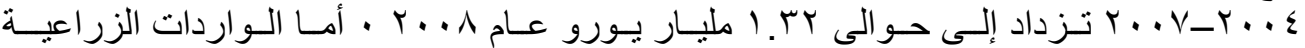

Fayoum J. Agric. Res. \& Dev., Vol.23, No.2, (B) July, 2009 
11.

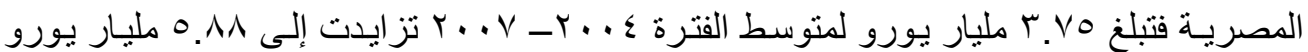

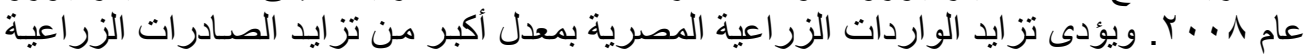

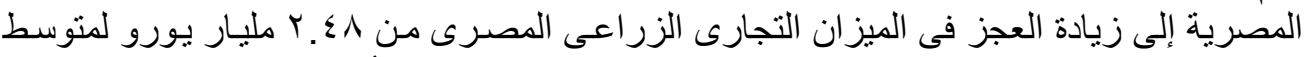

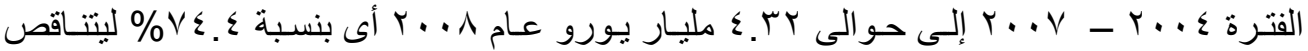

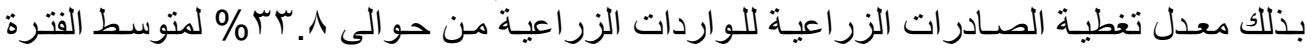

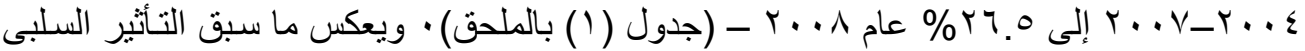

للأزمة المالية والاقتصادية العالمية على الميزان التجارى و الميزان التجارى الزراعى العى المصرى .

\section{التجارة الخارجية المصرية مع الاتحاد الأوربى:}

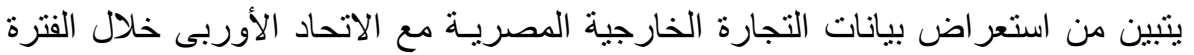

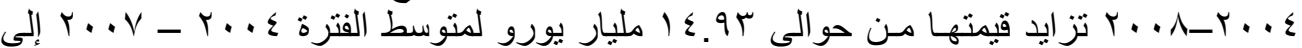

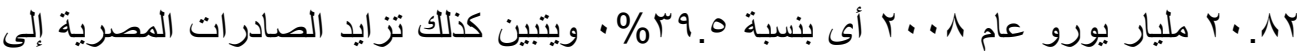

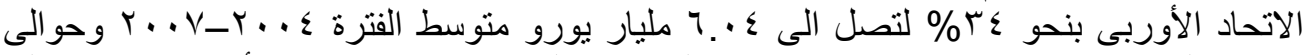

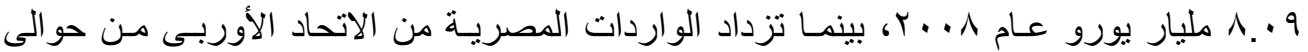

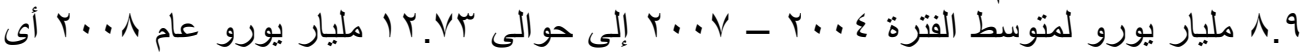

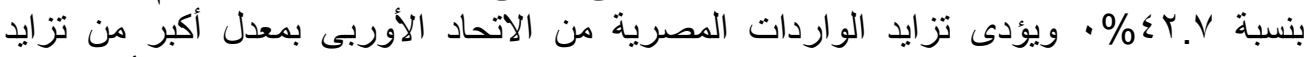

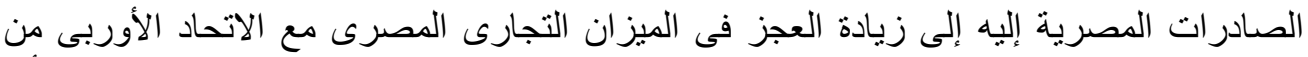

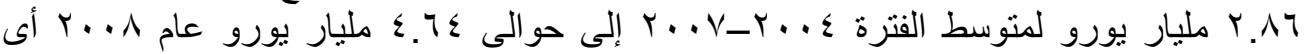

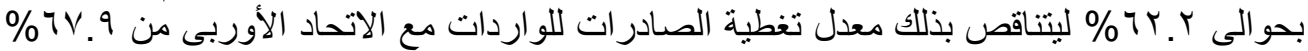

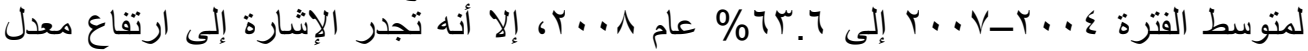

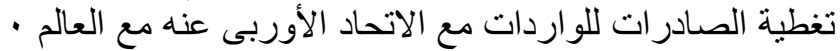

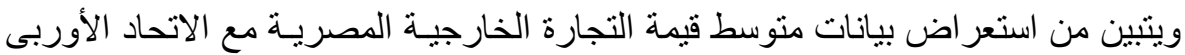

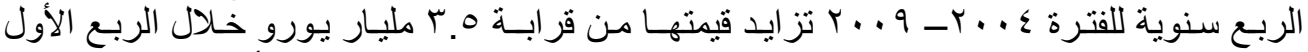

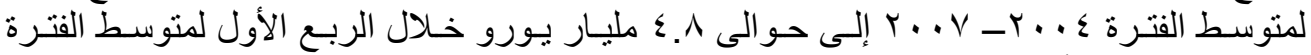

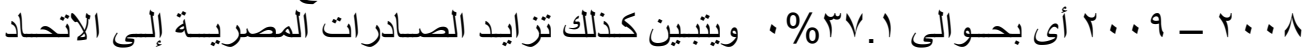

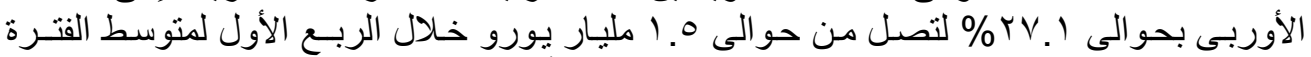

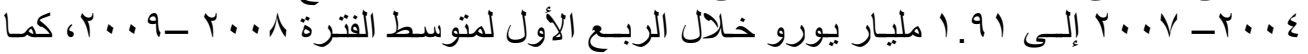

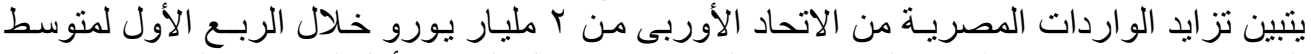

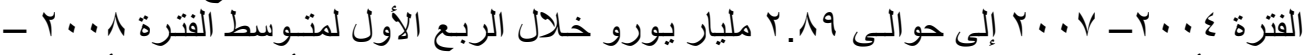

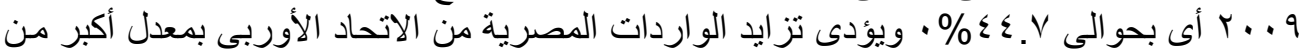

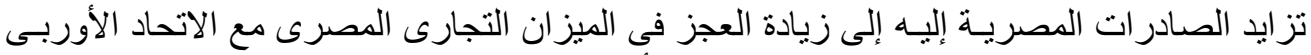

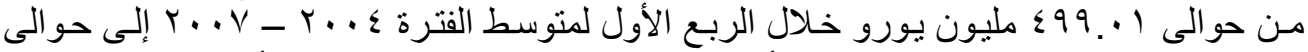

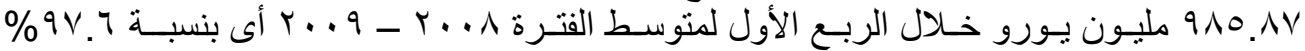

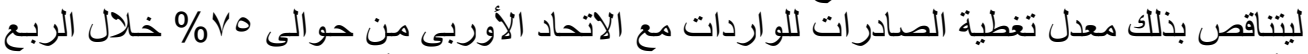

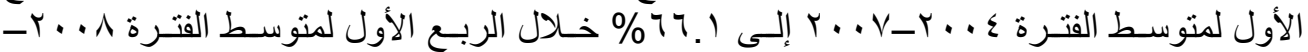

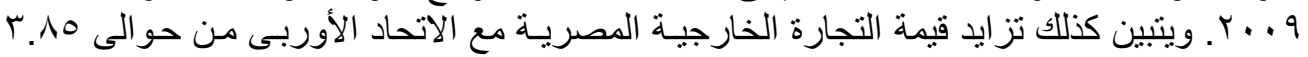

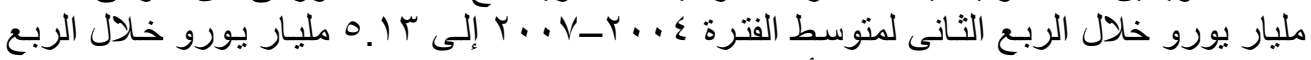

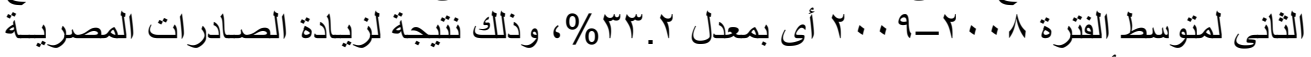

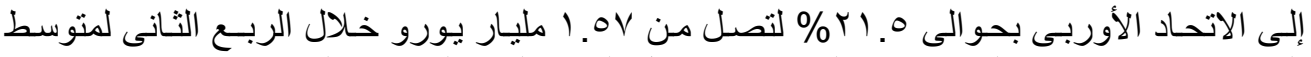

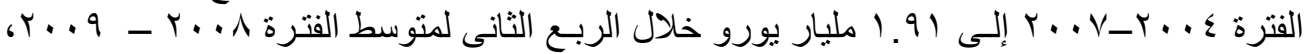

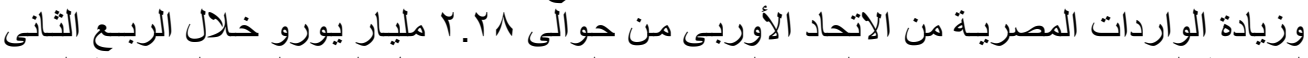

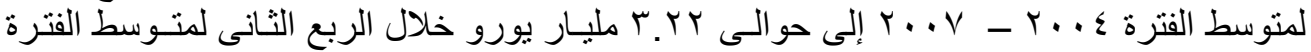

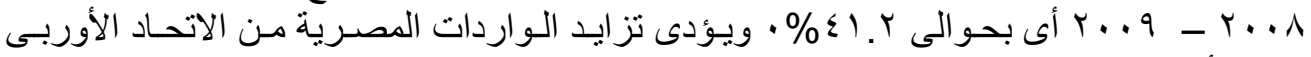

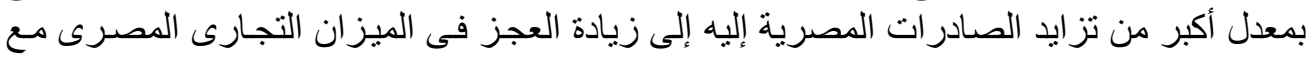

Fayoum J. Agric. Res. \& Dev., Vol.23, No.2, (B) July, 2009 
111

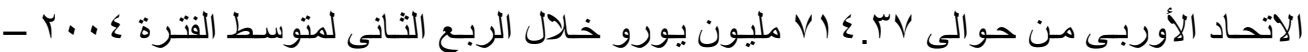

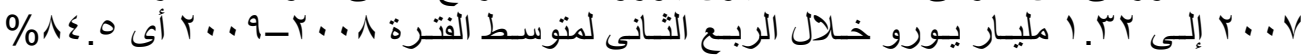

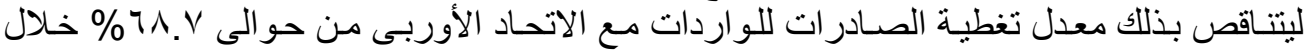

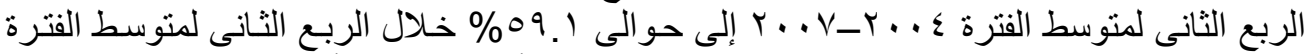

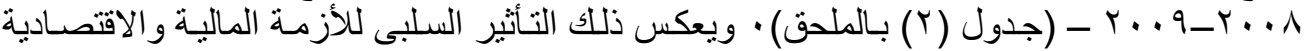

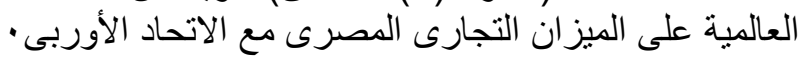
التجارة الخارجية الزراعية المصرية مع الاتحاد الأوربى:

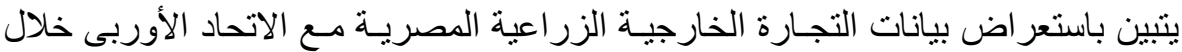

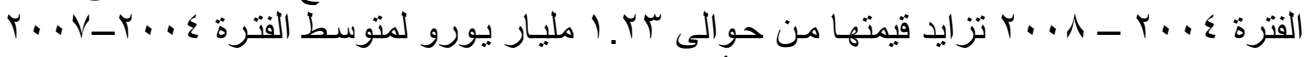

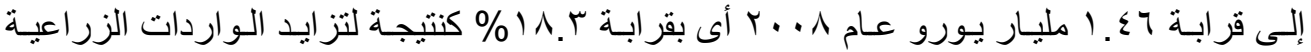

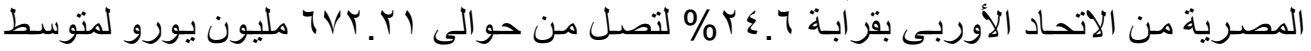

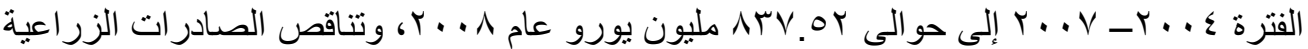

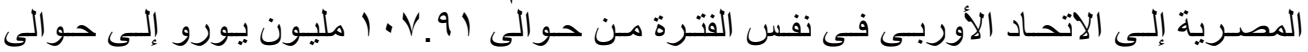

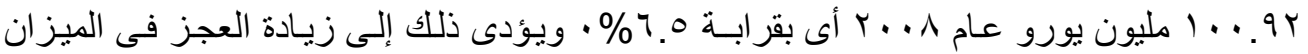

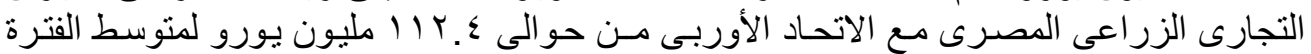

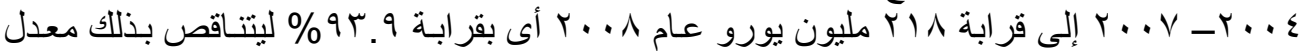

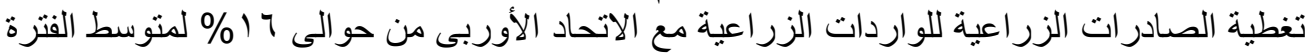

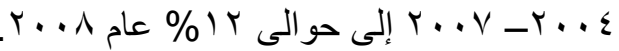

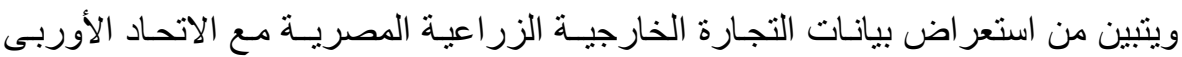

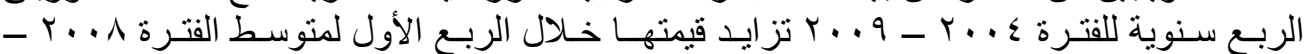

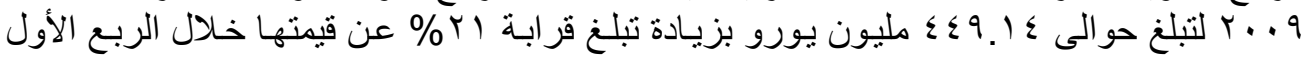

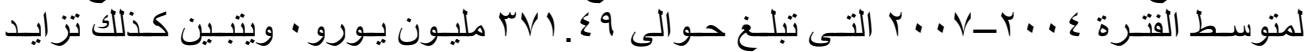

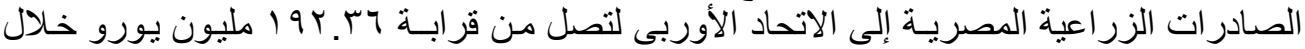

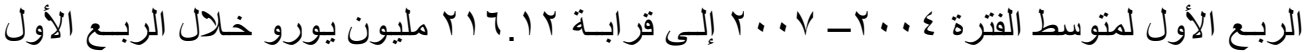

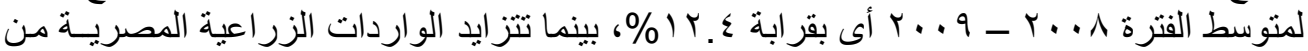

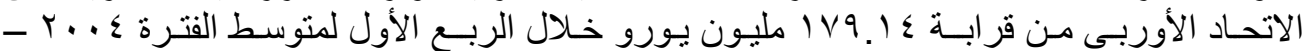

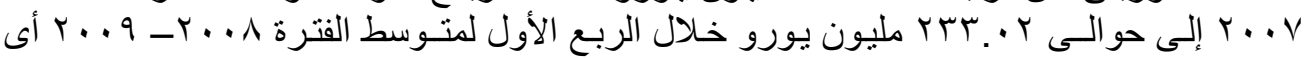

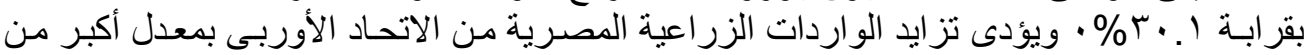

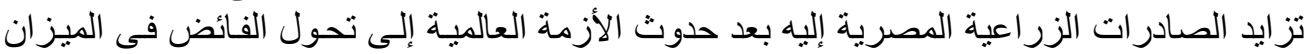

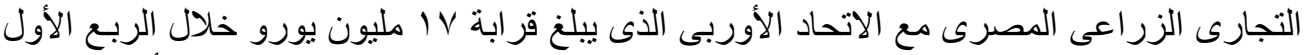

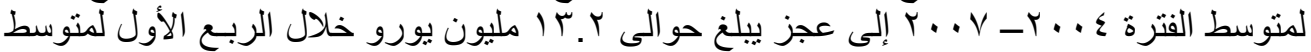

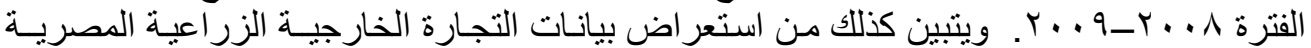

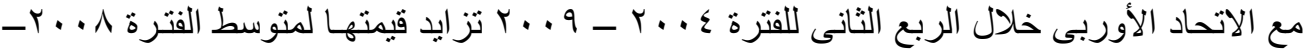

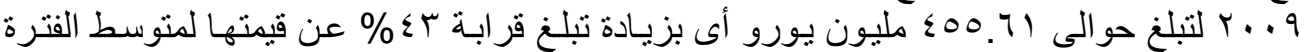

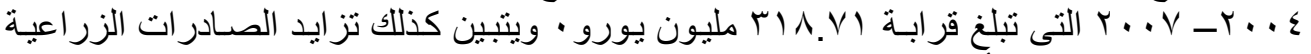

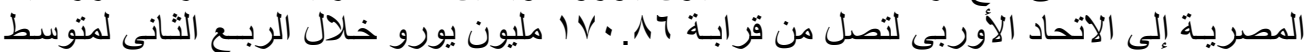

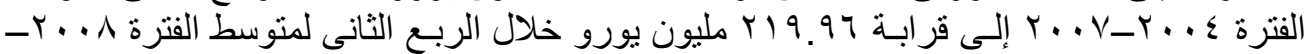

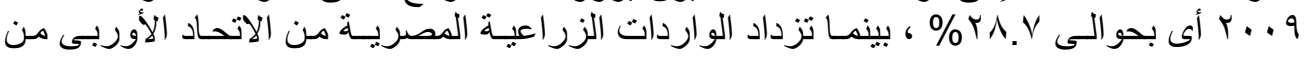

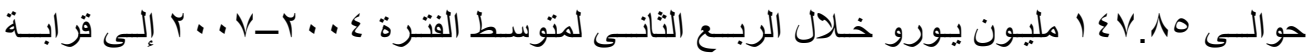

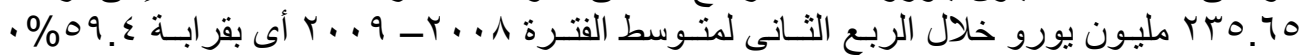

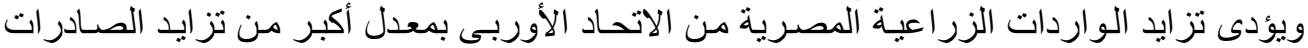

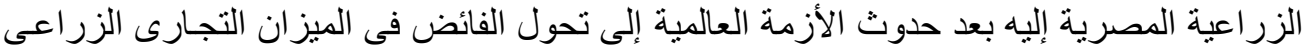

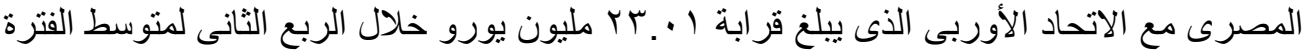

Fayoum J. Agric. Res. \& Dev., Vol.23, No.2, (B) July, 2009 


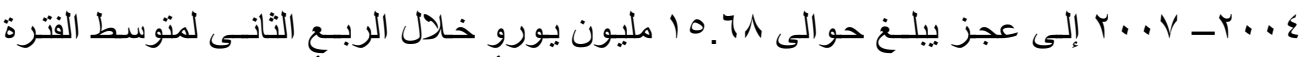

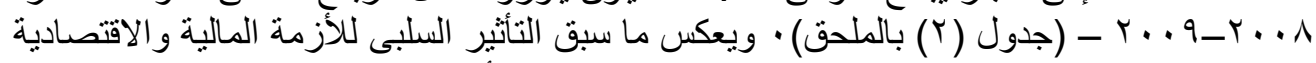

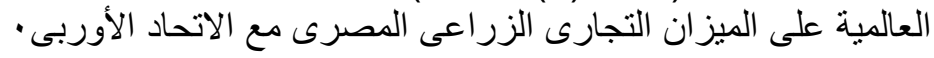
الصادرات الزراعية المصرية الرئيسية إلى الاتحاد الأوربى:

تمهيد : تجدر الإشارة إلى أن أهم الصادرات الزراعية المصرية إلى الاتحاد الأوربى على الترتيب

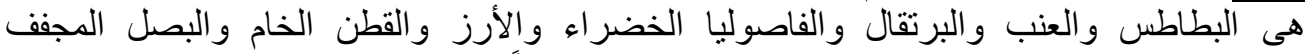

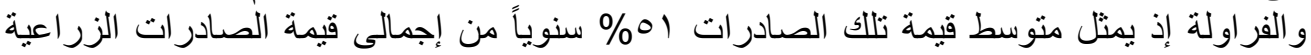

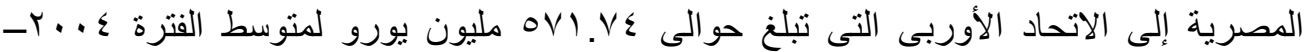

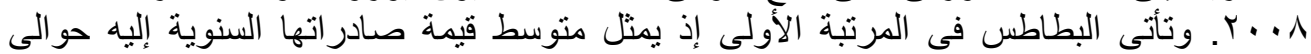

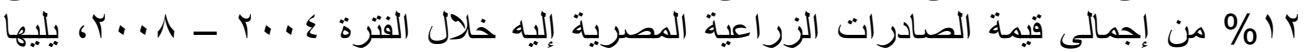

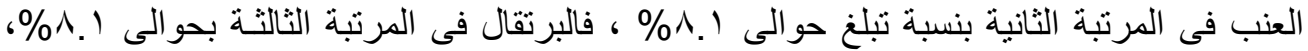

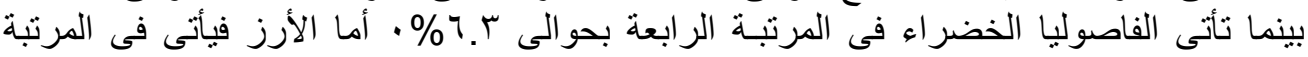

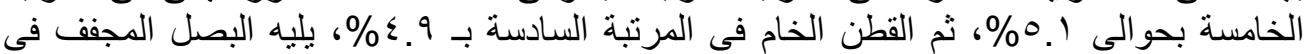

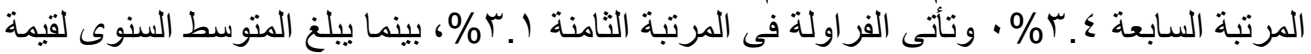

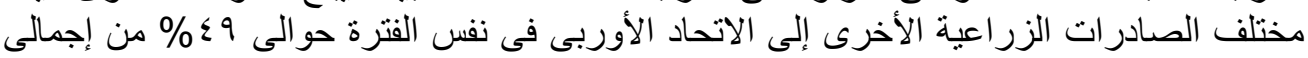

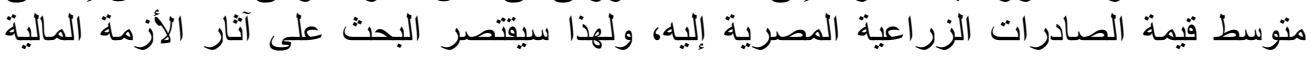

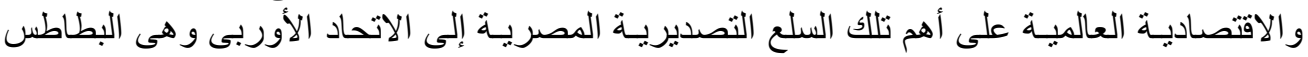

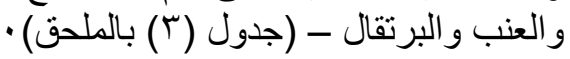

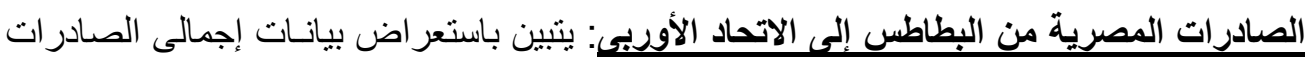

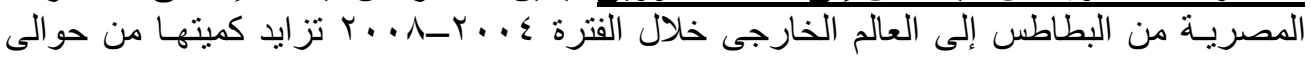

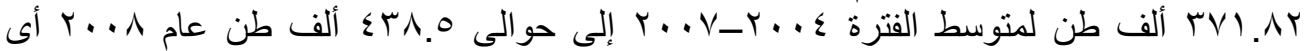

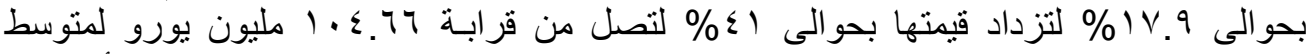

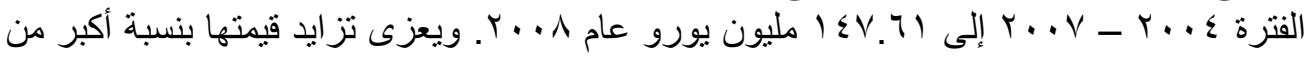

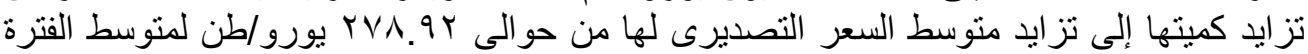

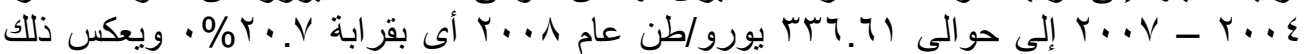

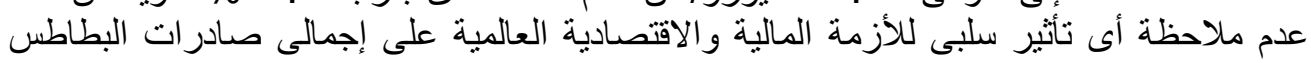

المصرية

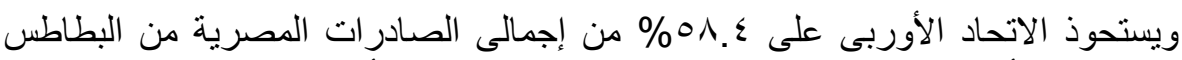

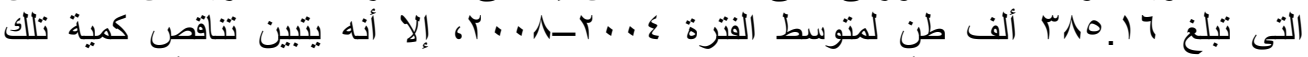

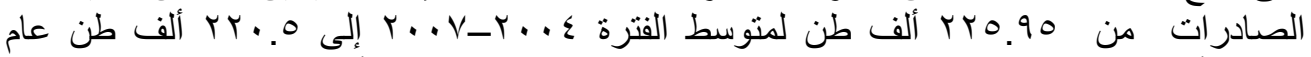

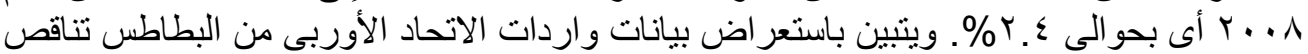

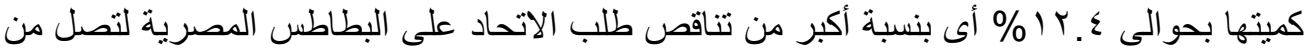

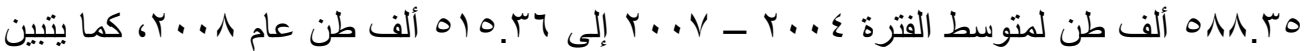

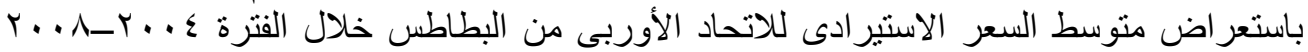

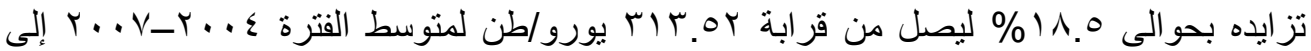

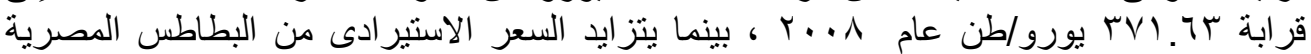

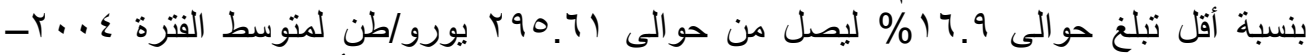

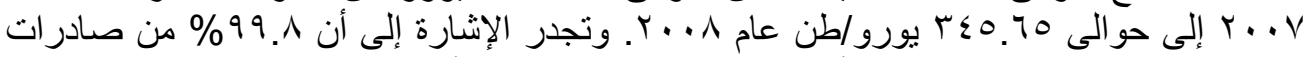

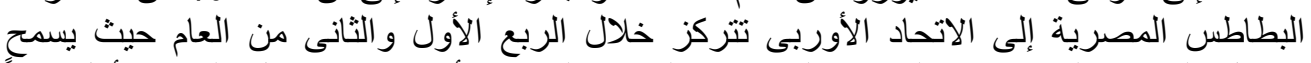

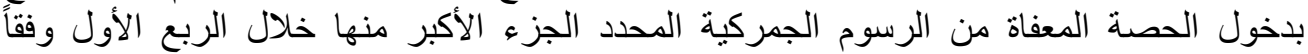

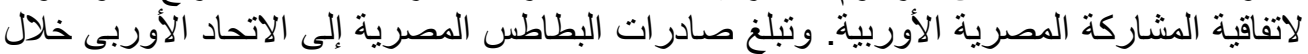

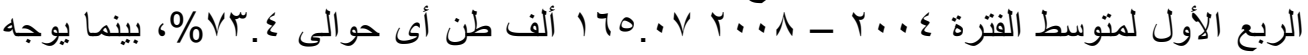

Fayoum J. Agric. Res. \& Dev., Vol.23, No.2, (B) July, 2009 
$11 \%$

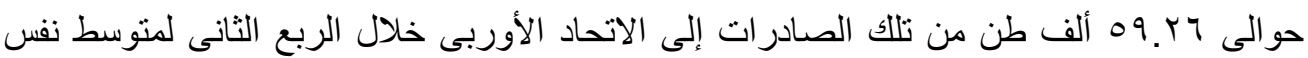

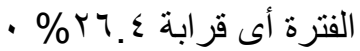

ويتبين باستعر اض بيانات الصادرات المصرية من البطاطس إلى الاتحاد الأوربى الربع سنوية البهابة

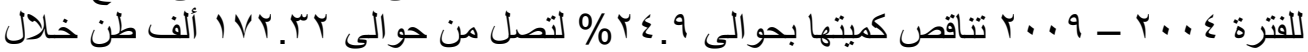

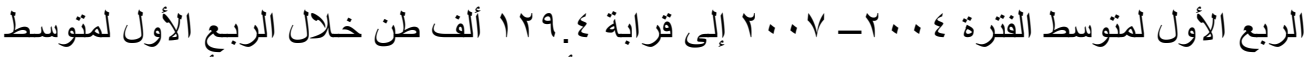

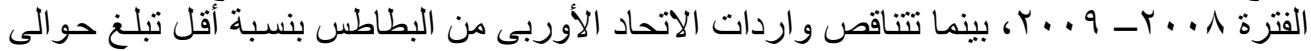

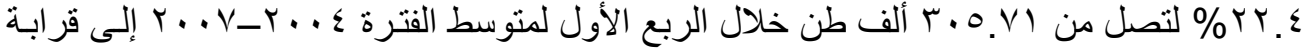

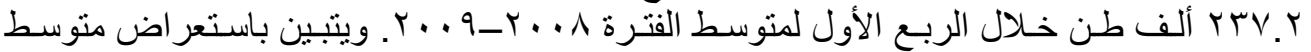

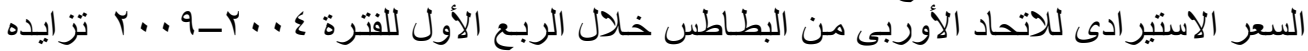

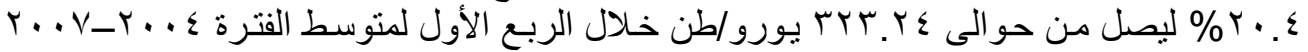

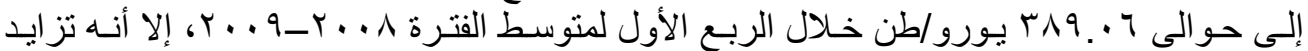

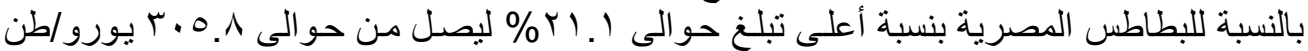

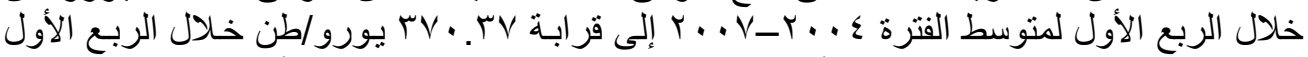

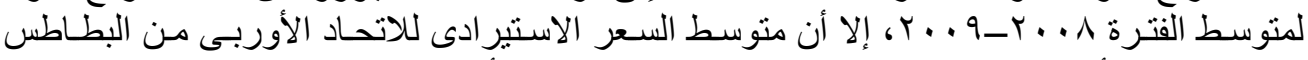

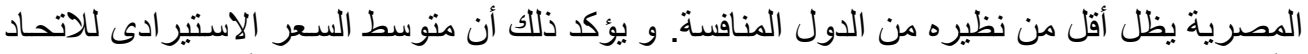

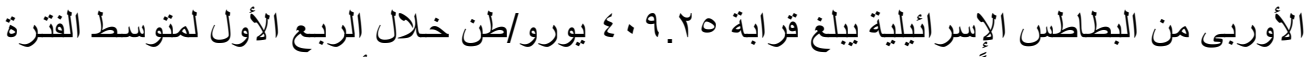

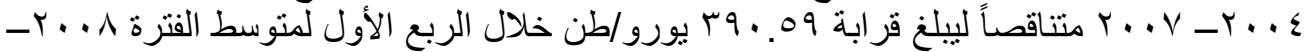

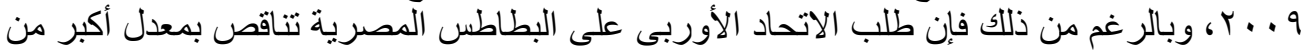

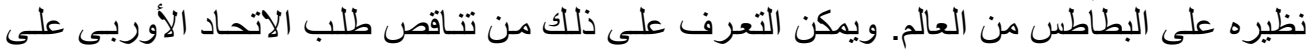

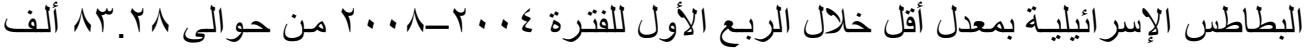

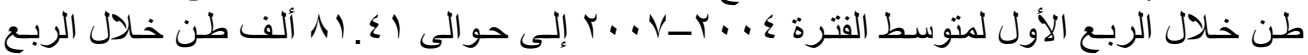

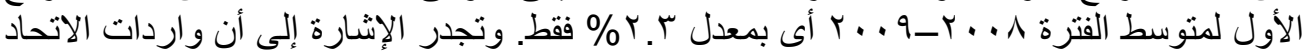

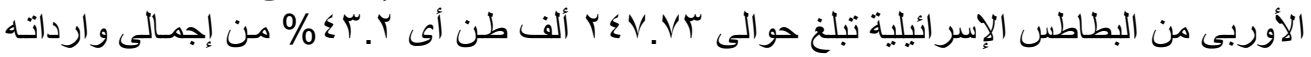

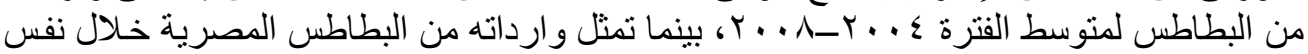

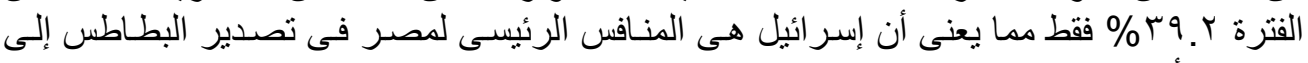
الاتحاد الأوربى.

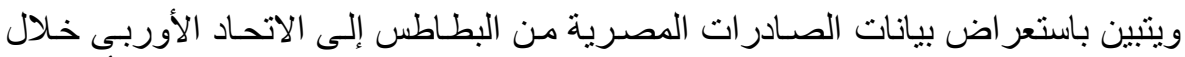

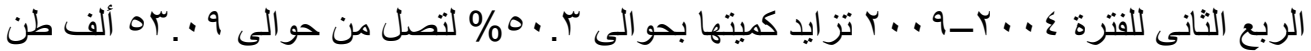

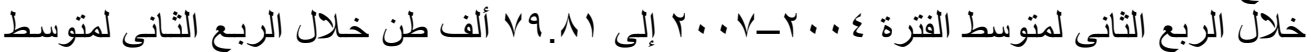

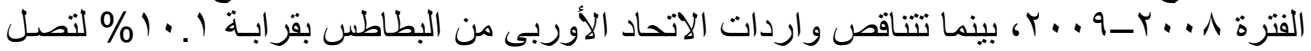

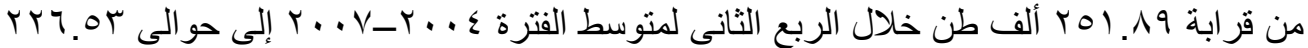

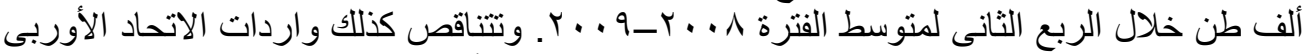

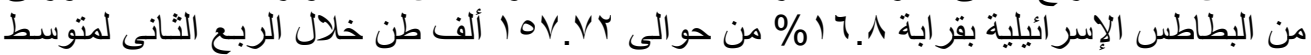

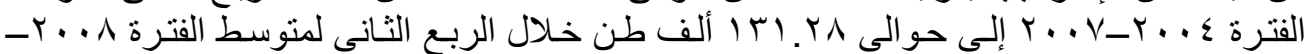

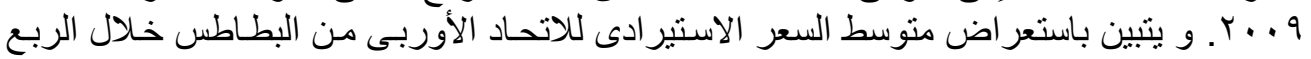

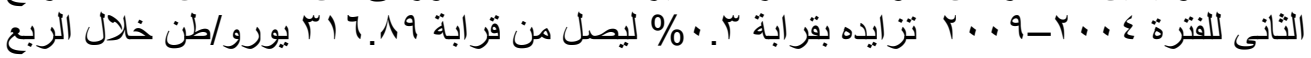

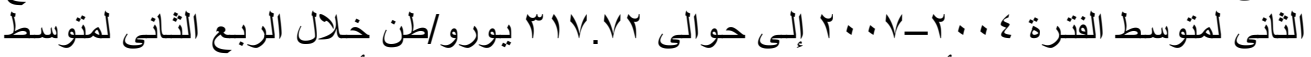

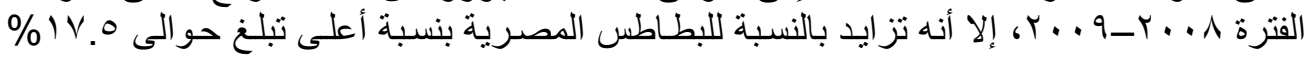

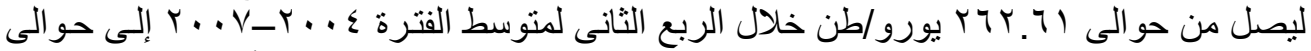

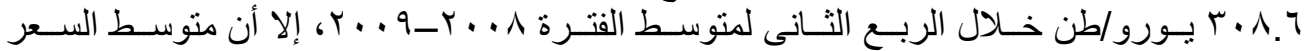

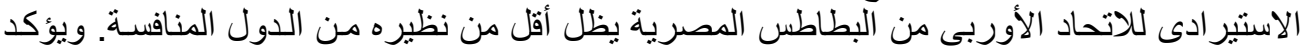

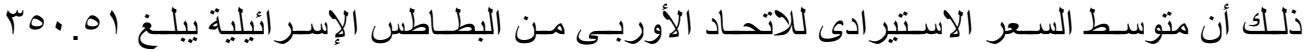

Fayoum J. Agric. Res. \& Dev., Vol.23, No.2, (B) July, 2009 
$11 \varepsilon$

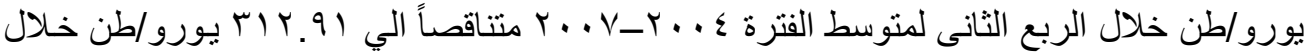

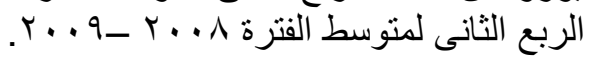

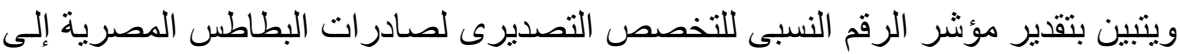

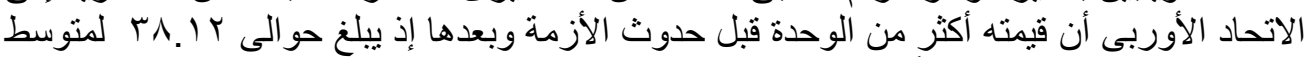

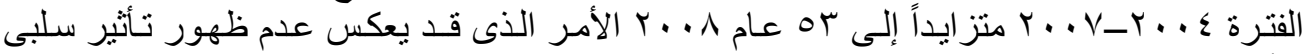

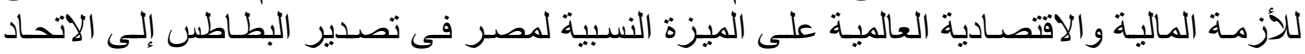

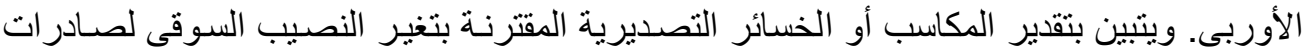

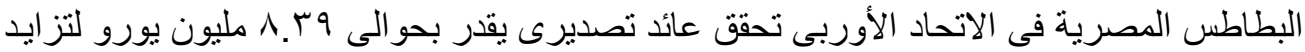

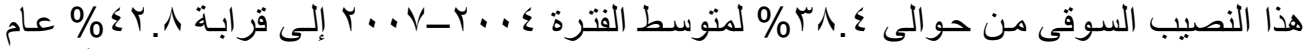

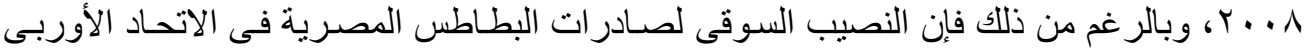

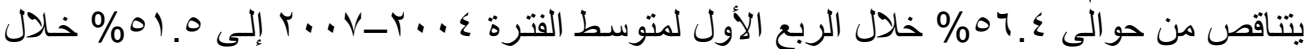

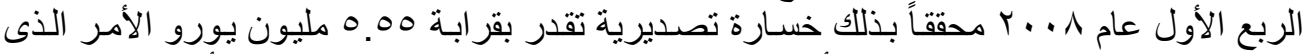

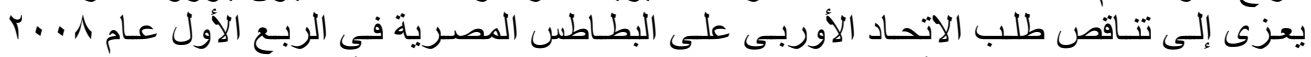

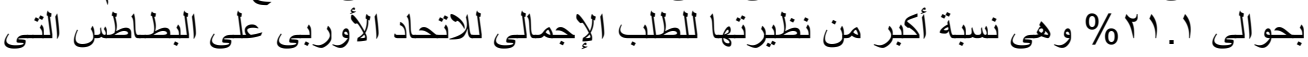

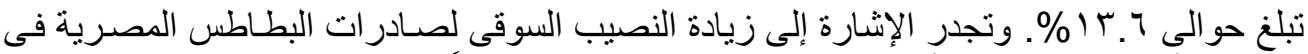

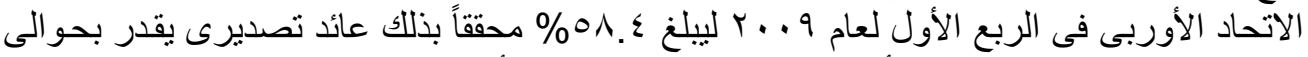

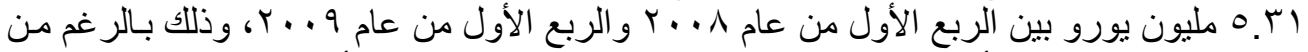

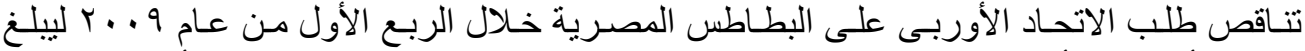

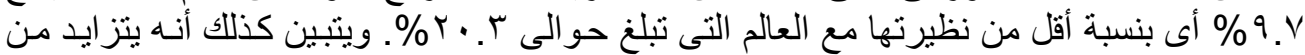

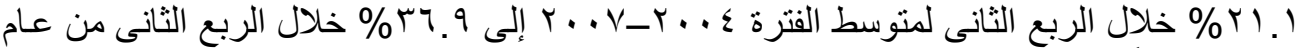

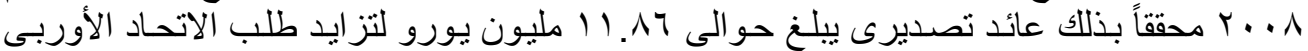

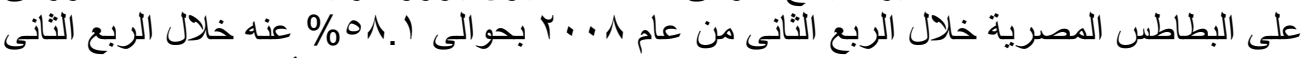

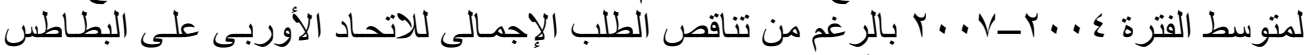

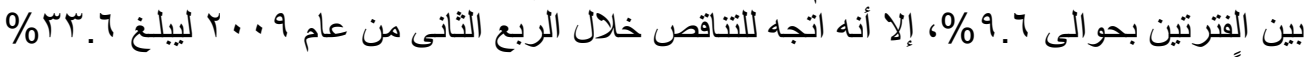

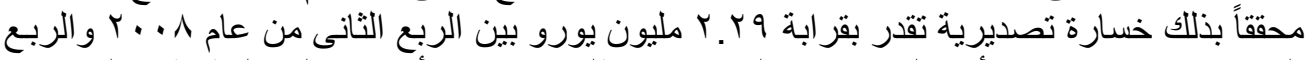

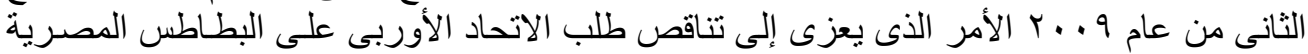

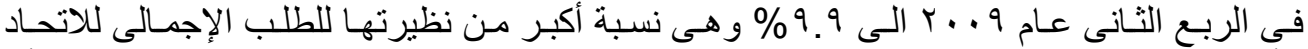

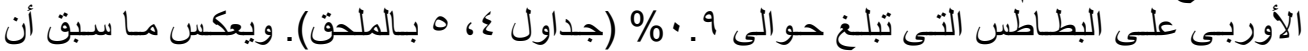

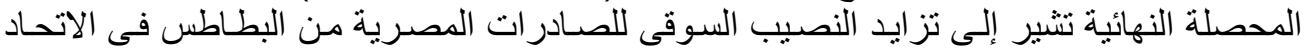

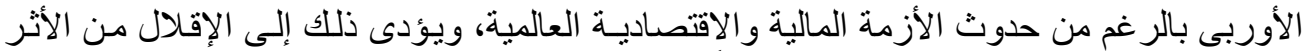

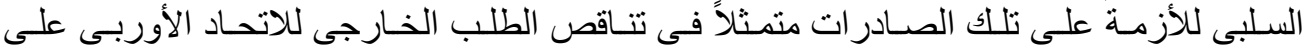

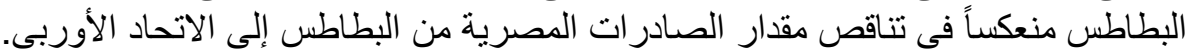

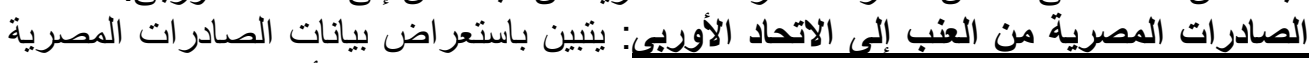

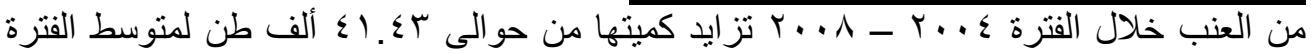

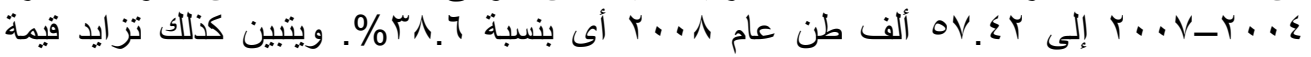

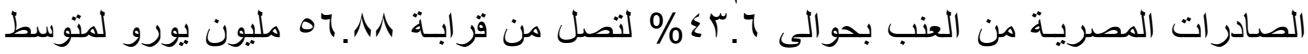

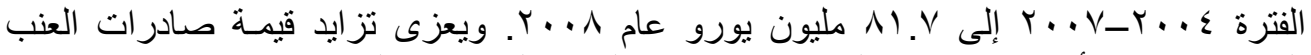

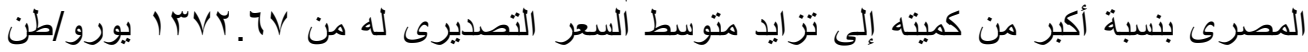

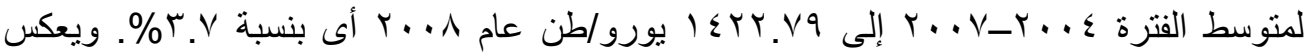

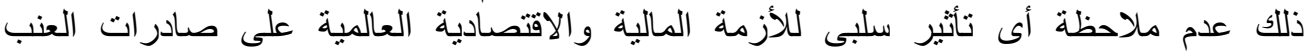

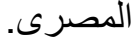

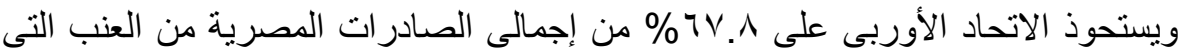

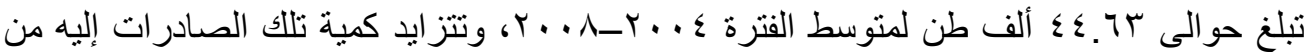

Fayoum J. Agric. Res. \& Dev., Vol.23, No.2, (B) July, 2009 
110

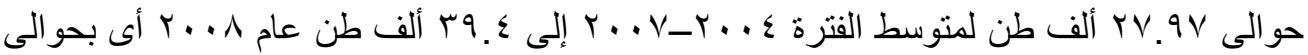

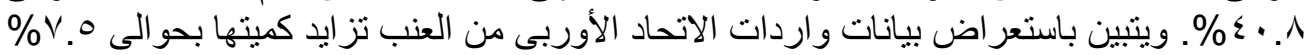

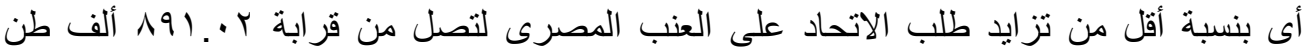

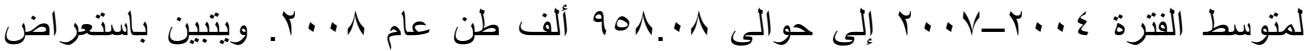

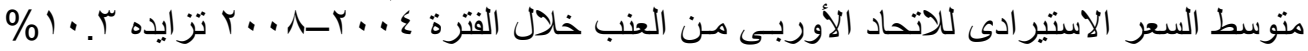

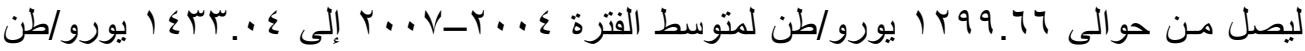

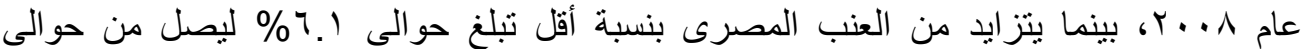

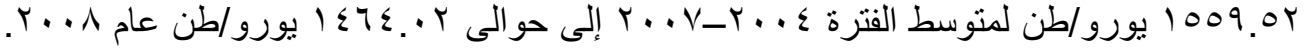
وتجدر الإشارة إلى أن حوالى 9.9 9\% من صادر ات الت العنب المصرى إلى الاتحاد الأوربى تتركز

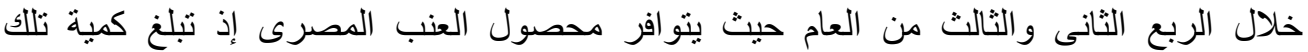

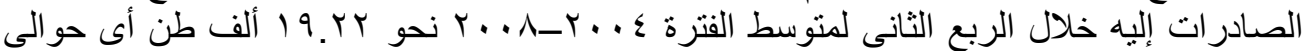

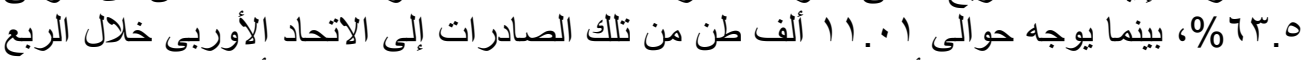

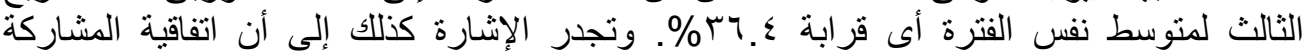

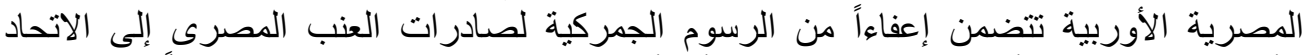

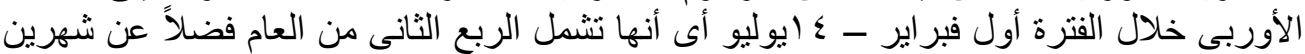

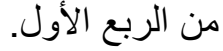

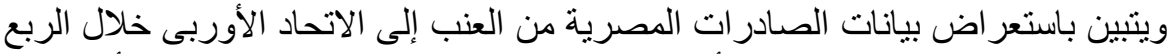

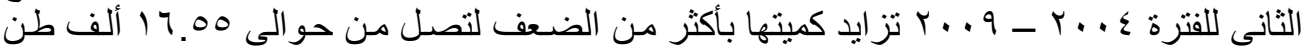

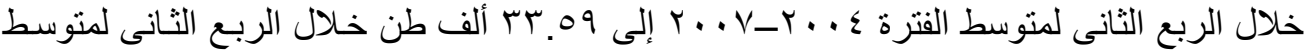

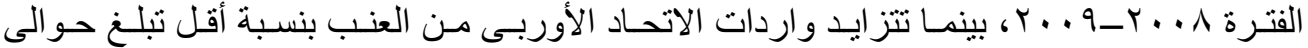

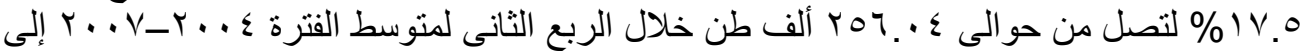

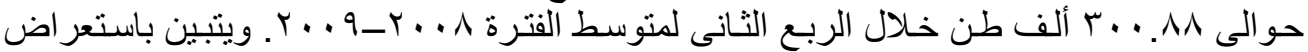

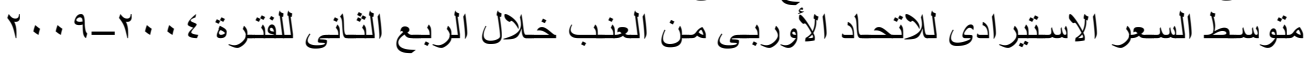

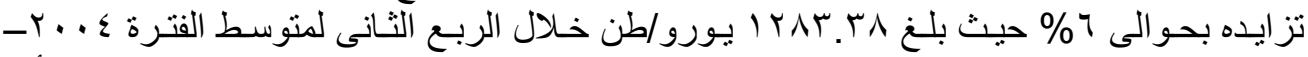

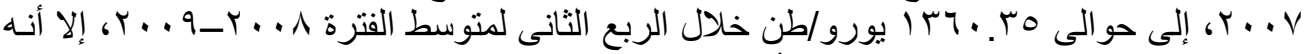

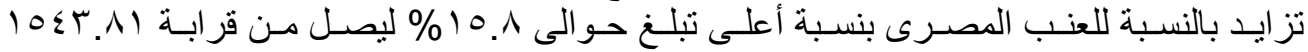

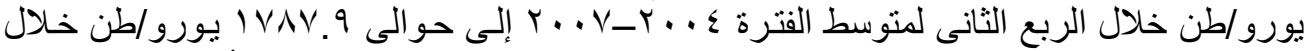

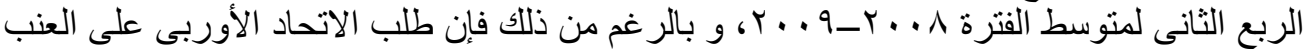

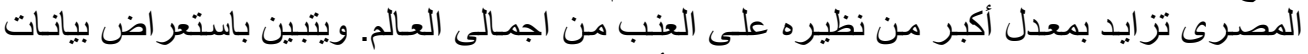

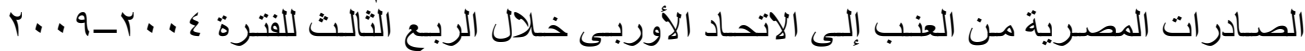

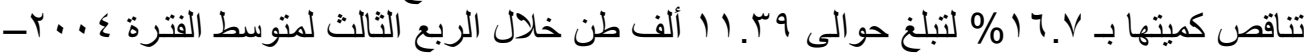

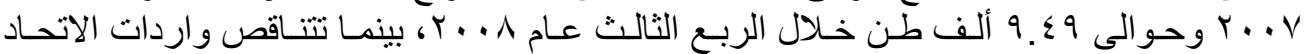

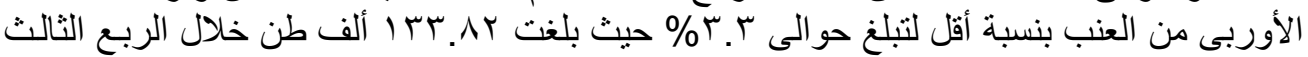

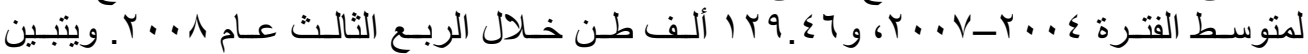

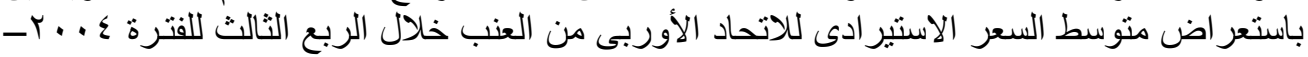

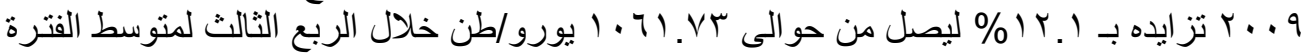

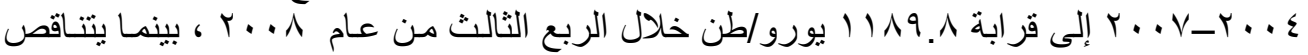

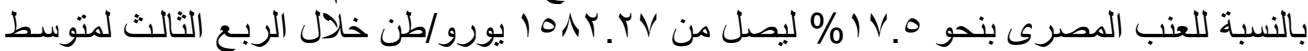

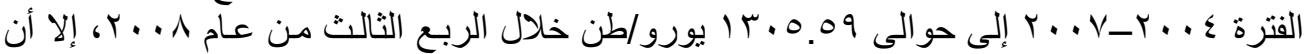

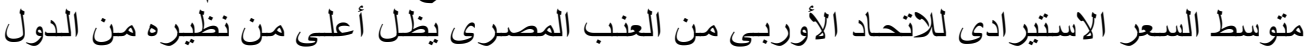
المنافسة

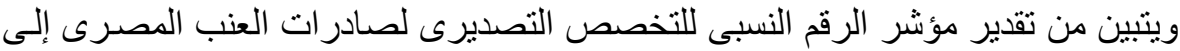

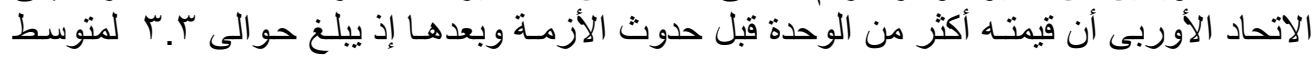

Fayoum J. Agric. Res. \& Dev., Vol.23, No.2, (B) July, 2009 
119

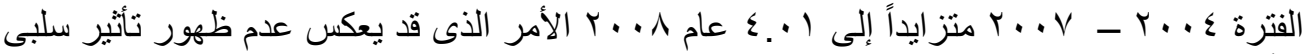

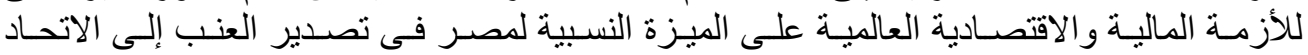

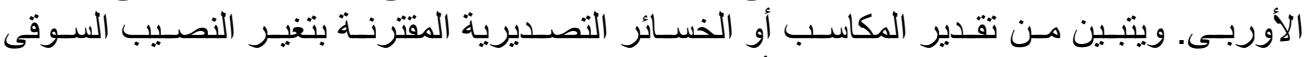

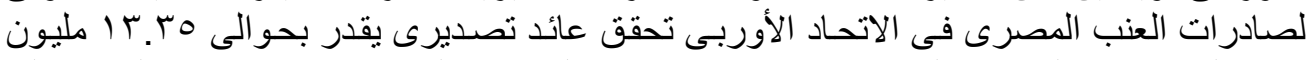

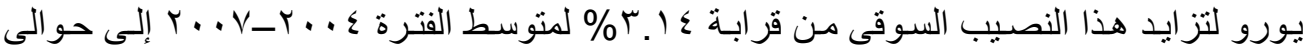

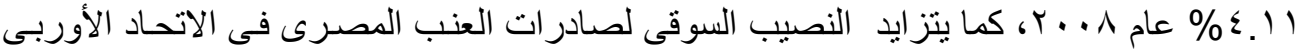

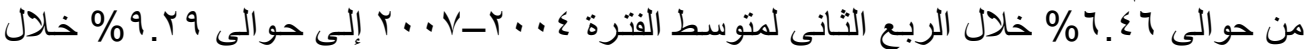

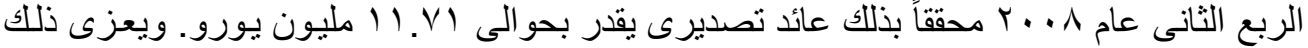

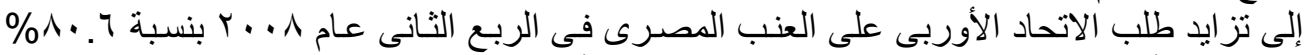

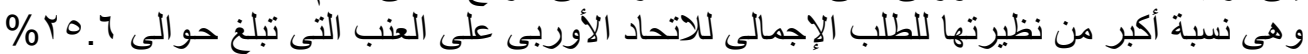

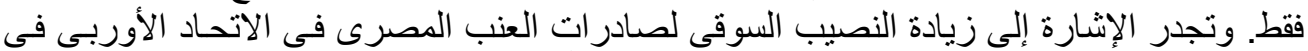

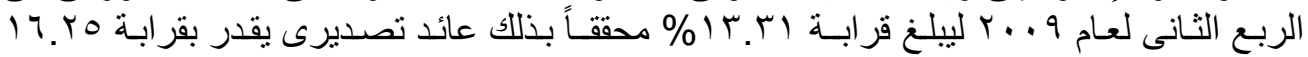

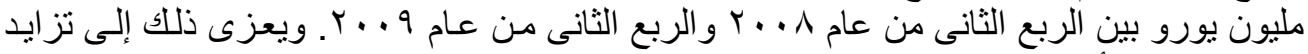

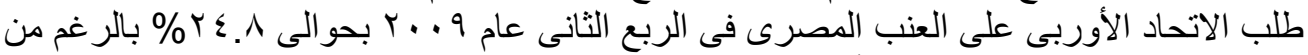

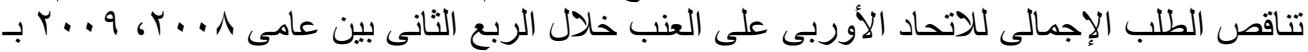

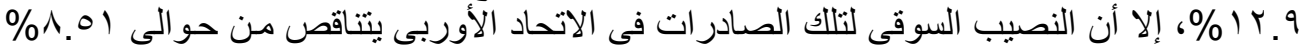

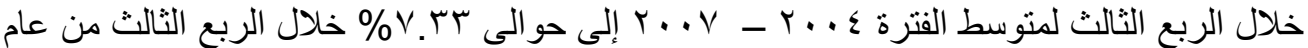

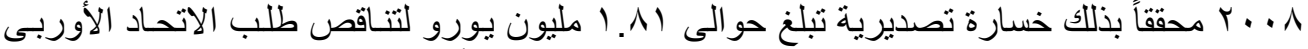

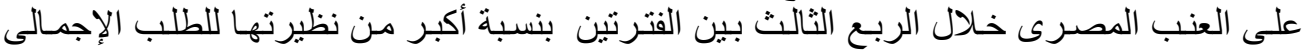

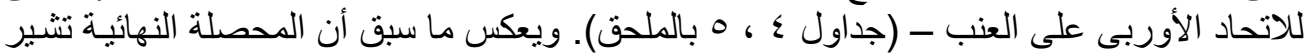

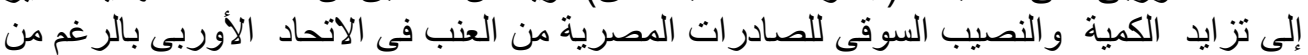
حدوث الأزمة المالية و الاقتصادية.

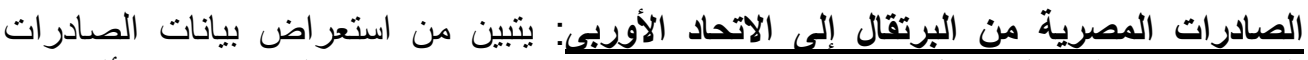

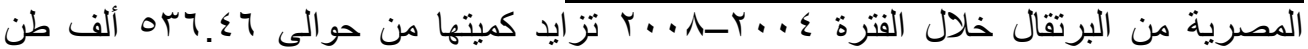

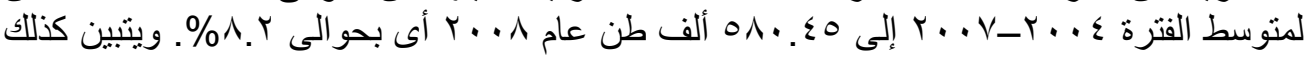

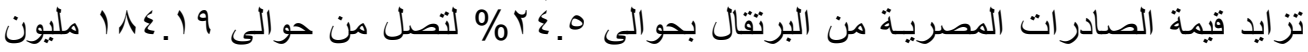

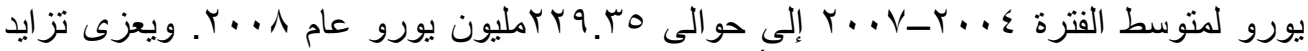

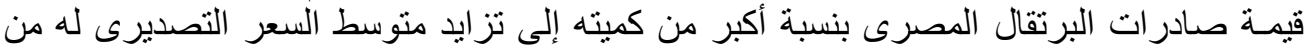

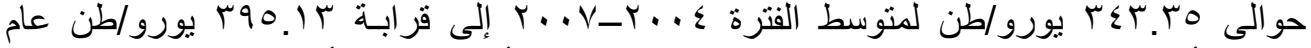

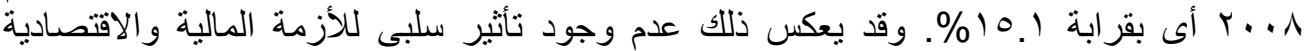
العالمية على صادر ات البرتقال المصرى.

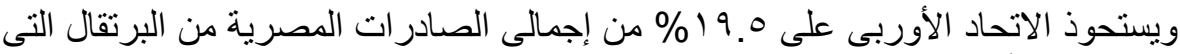

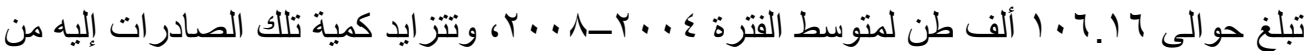

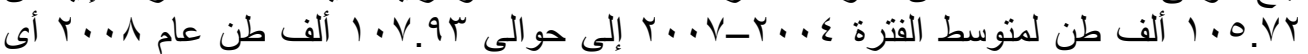

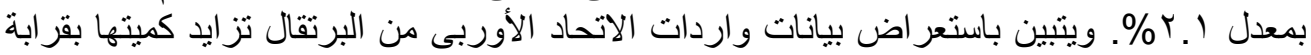

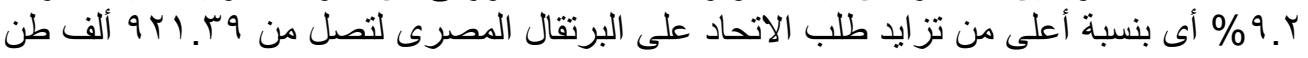

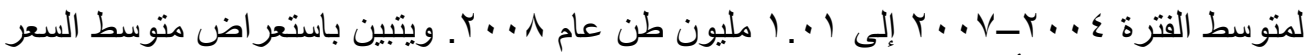

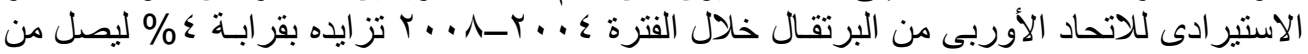

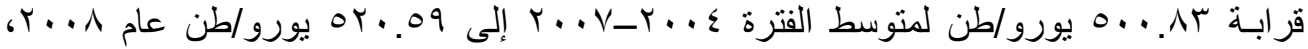

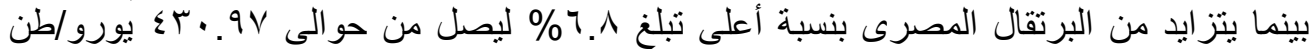

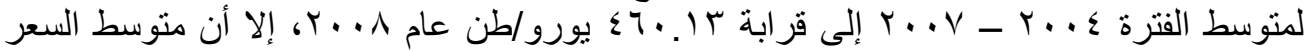

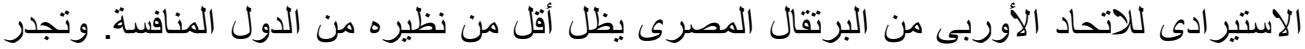

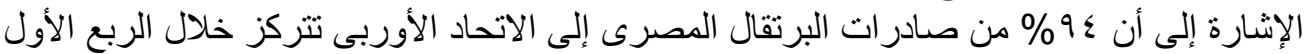

Fayoum J. Agric. Res. \& Dev., Vol.23, No.2, (B) July, 2009 
$11 \mathrm{~V}$

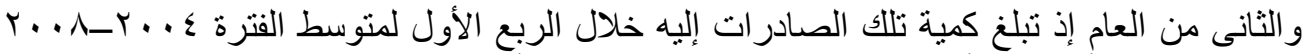

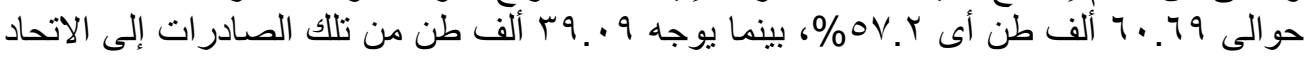

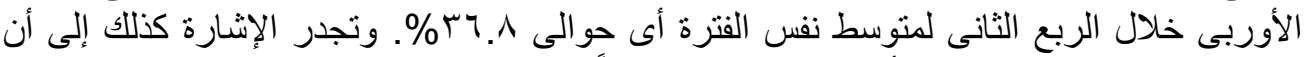

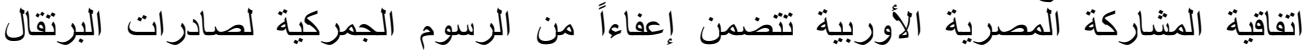

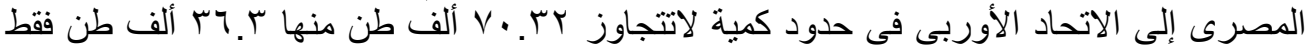

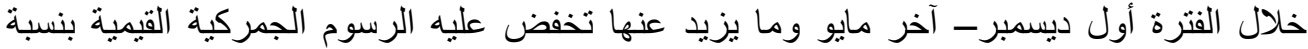

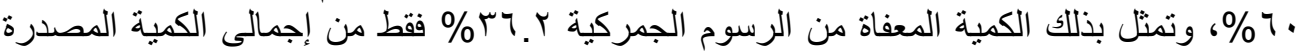

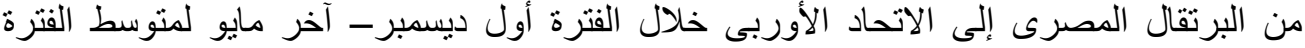

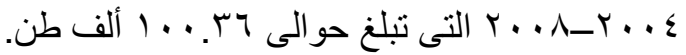

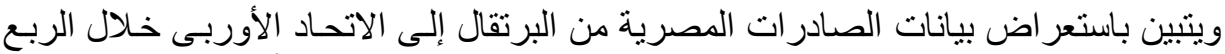

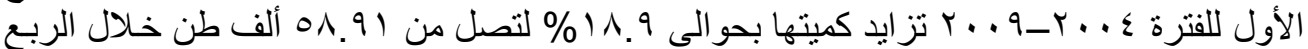

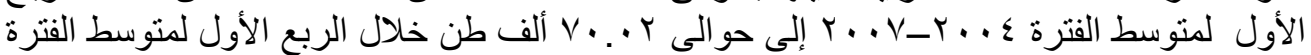

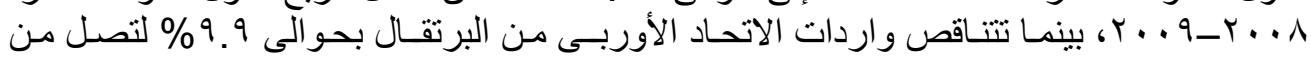

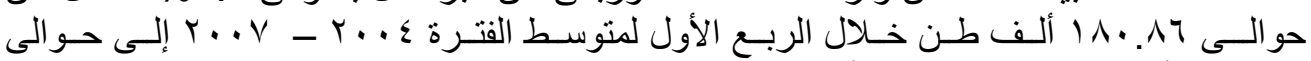

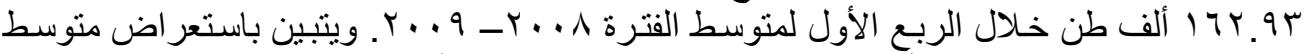

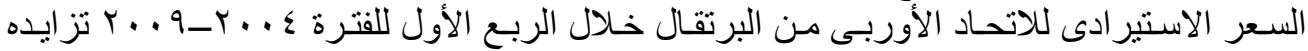

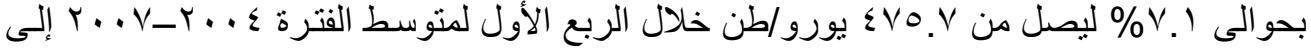

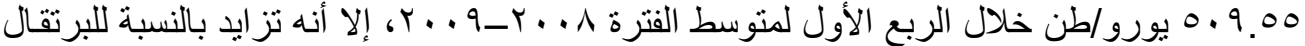

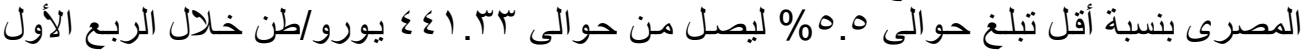

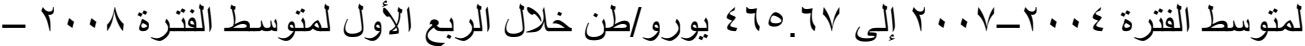

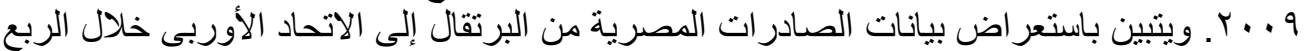

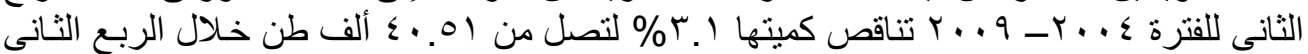

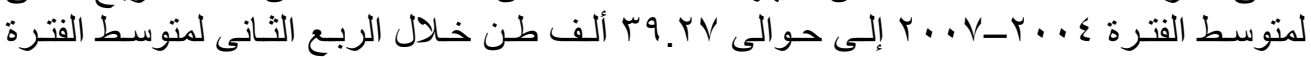

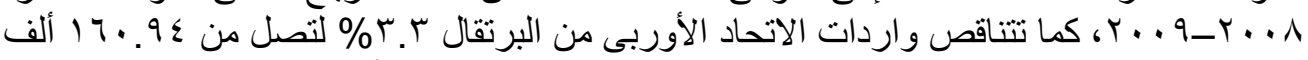

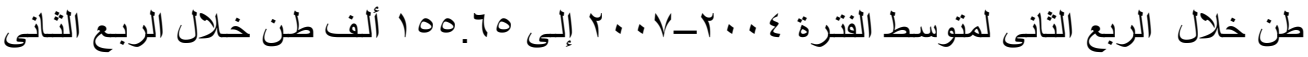

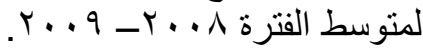

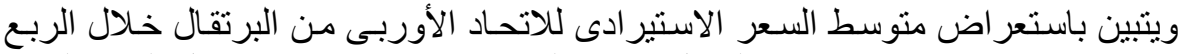

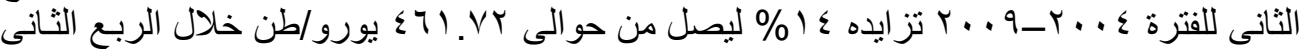

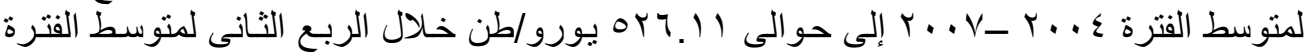

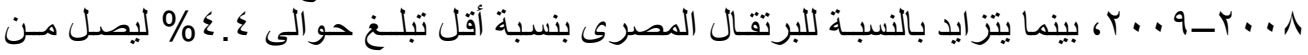

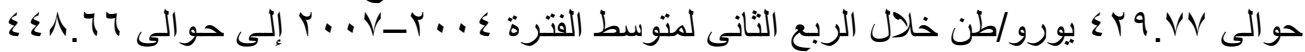

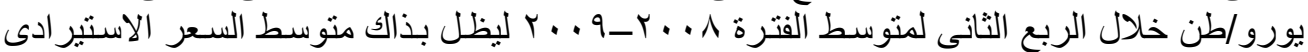

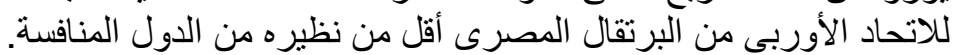

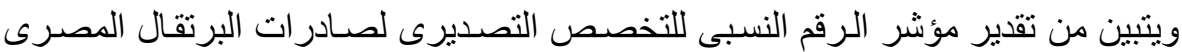

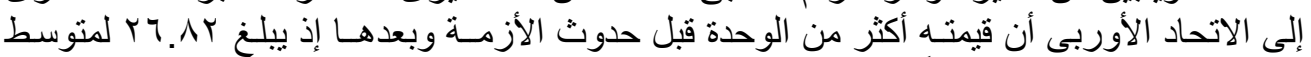

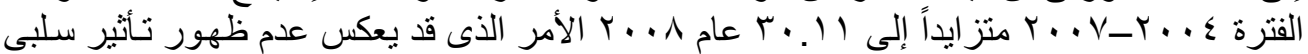

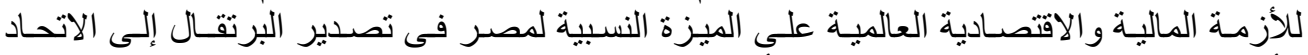

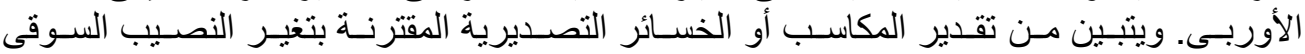

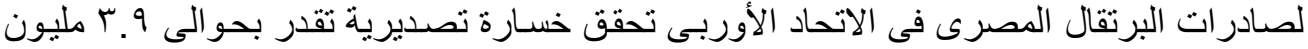

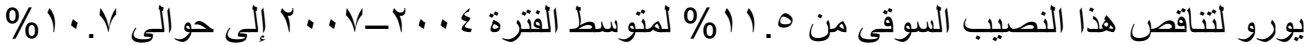

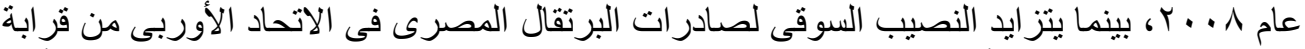

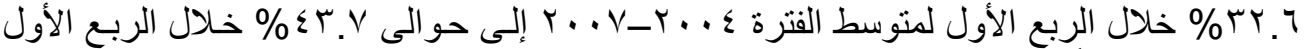

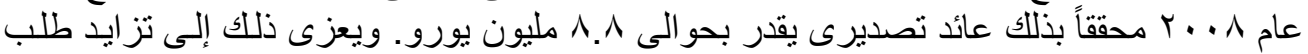

Fayoum J. Agric. Res. \& Dev., Vol.23, No.2, (B) July, 2009 
111

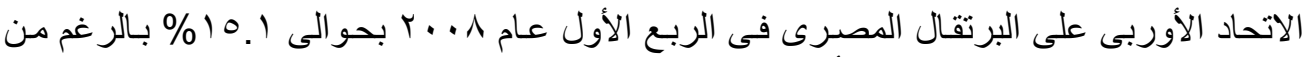

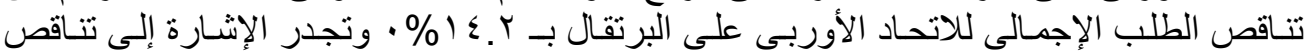

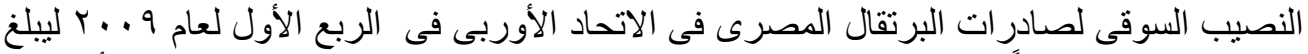

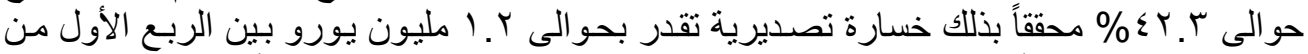

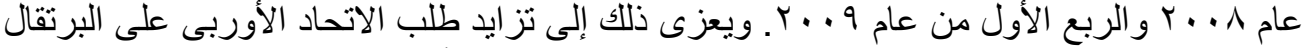

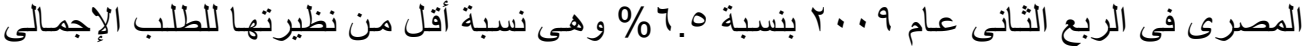

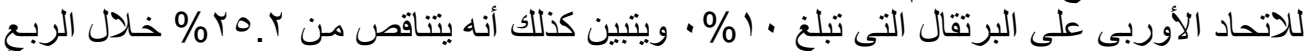

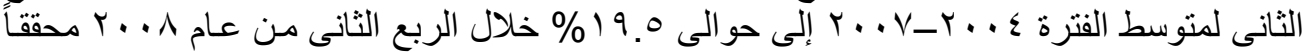

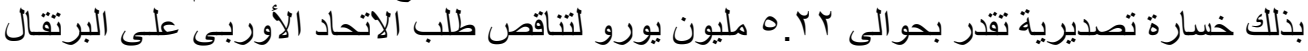

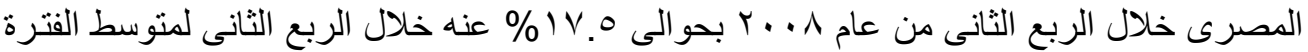

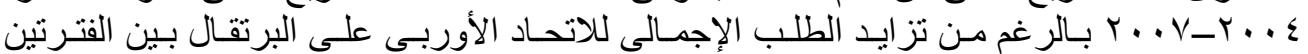

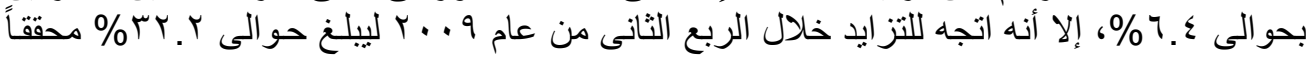

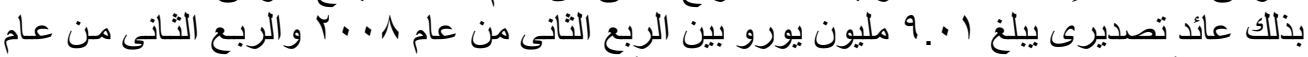

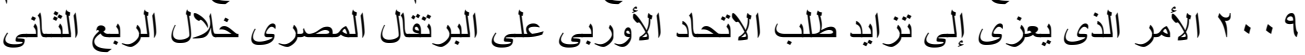

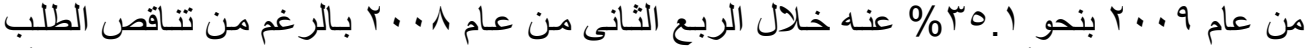

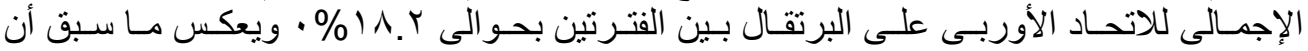

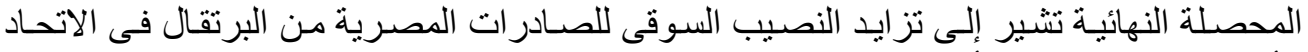

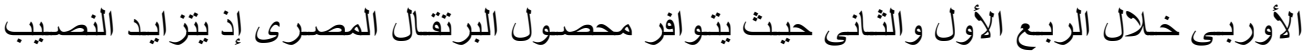

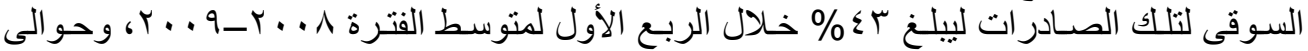

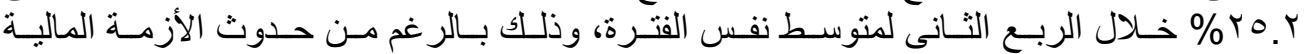

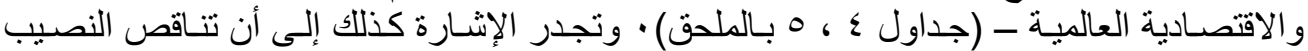

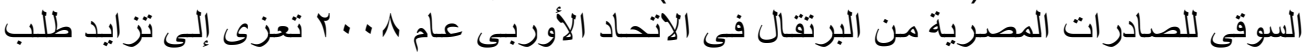

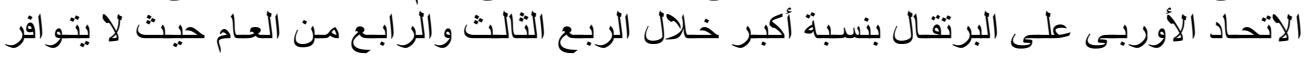

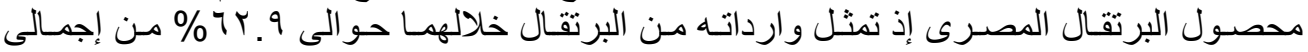

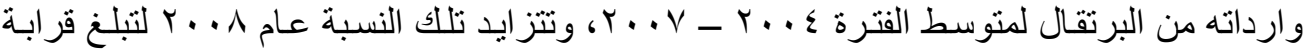

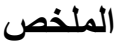

\% \% . .

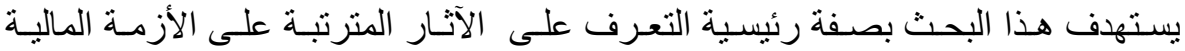

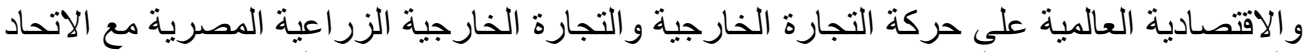

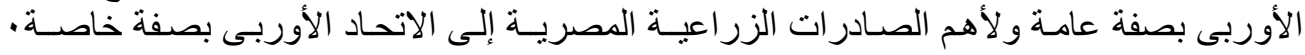

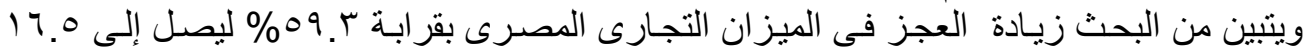

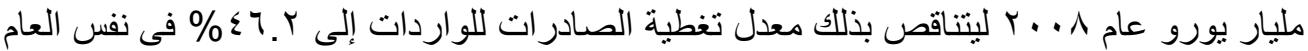

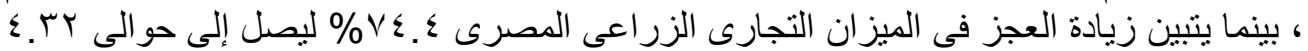

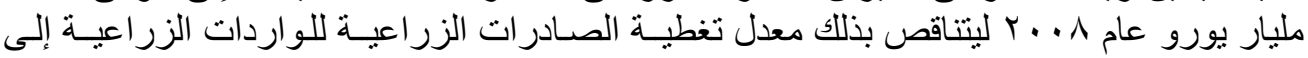

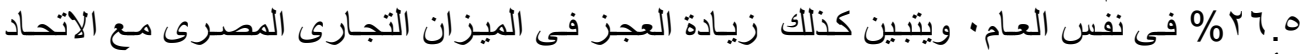

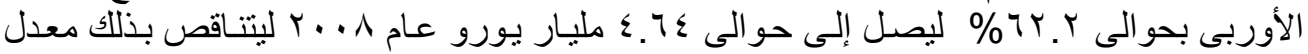

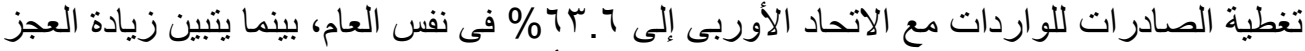

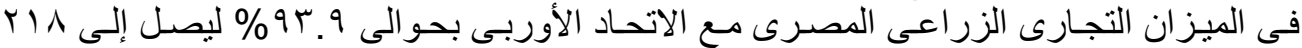

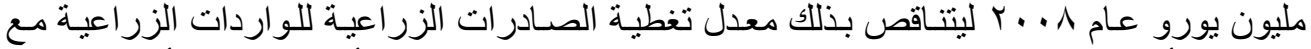

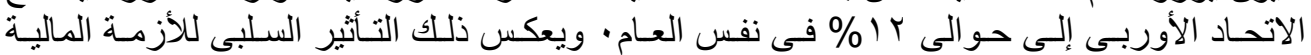

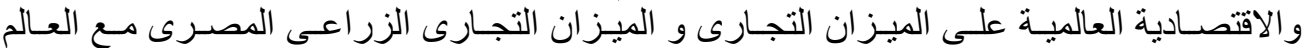

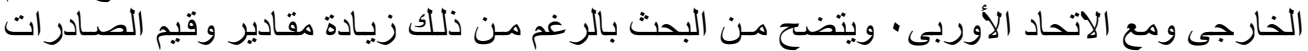

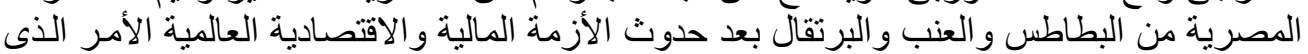

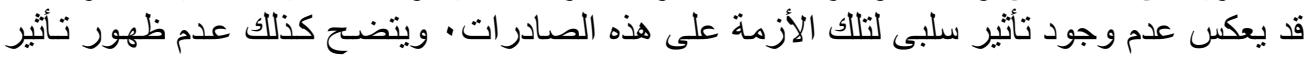

Fayoum J. Agric. Res. \& Dev., Vol.23, No.2, (B) July, 2009 
119

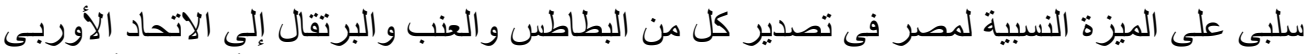

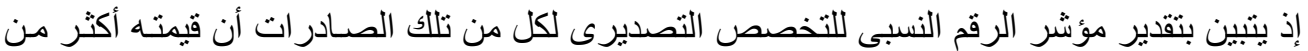

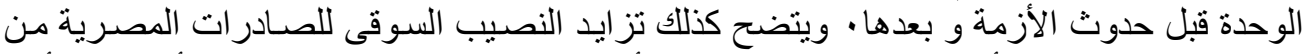

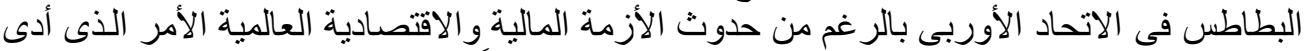

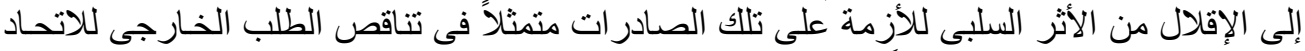

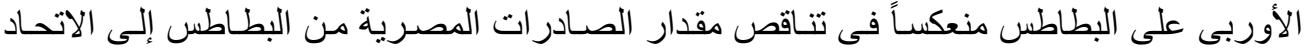

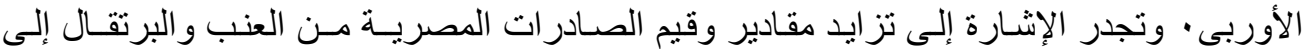

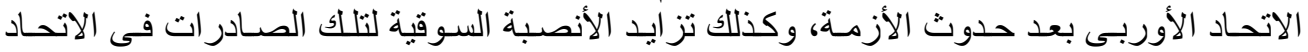

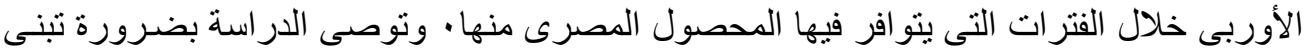

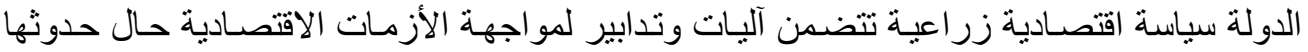

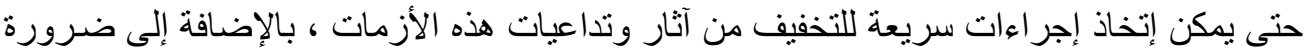

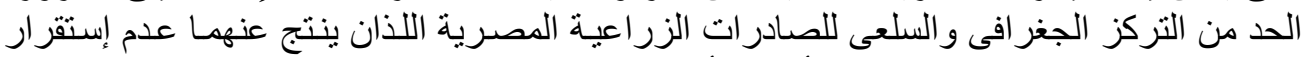

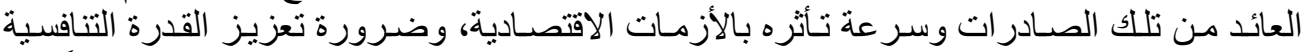

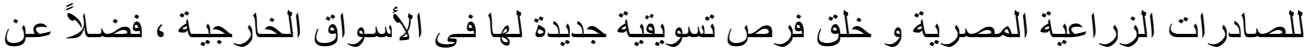

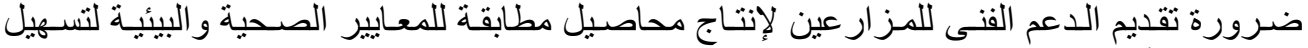

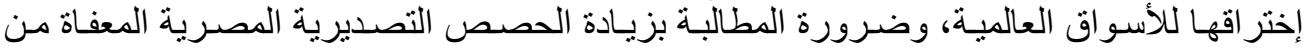
الجمارك إلى الاتحاد الأوربى وتعديل مو اسمها بما يتلاءم مع مواسم إنتاجها فى مصر •

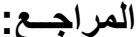

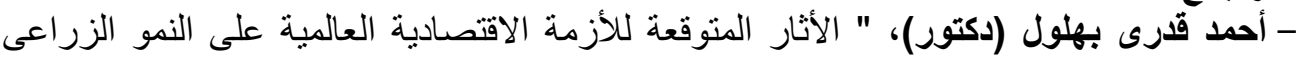

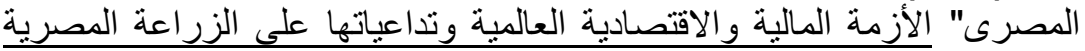

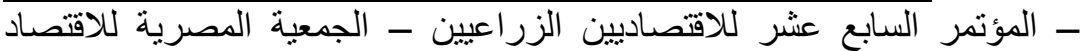

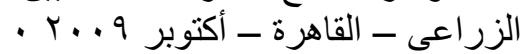

- أسامة أحمد البهنساوى (دكتور)، "دور السياسة الزراعية فى مواجهة تداعيات الأزمة المالية

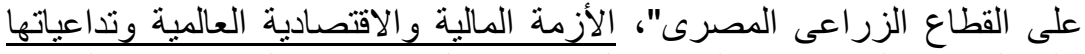

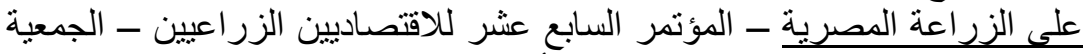

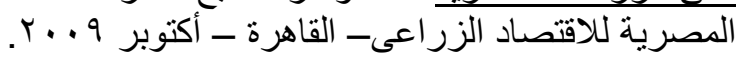

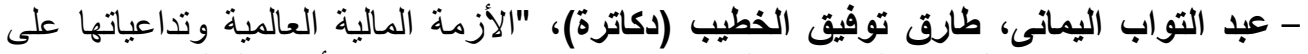

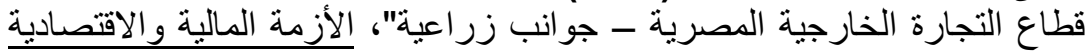

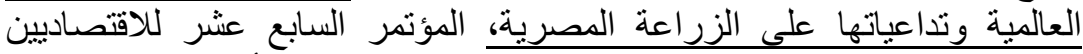

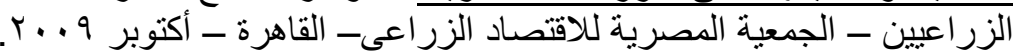

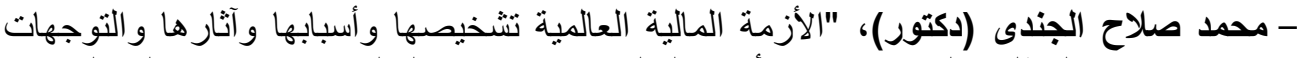

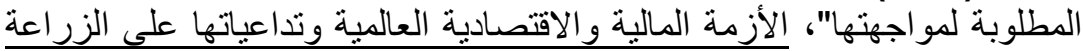

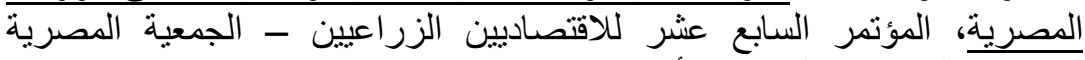

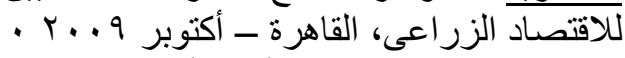

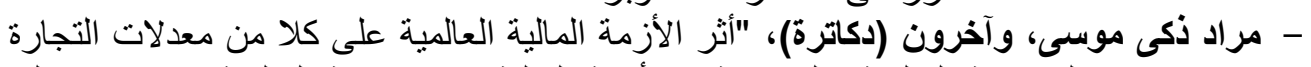

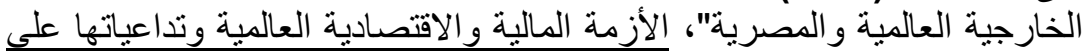

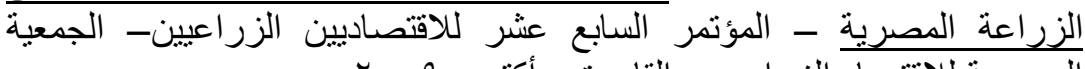

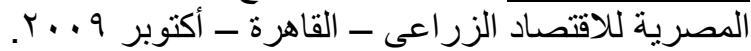

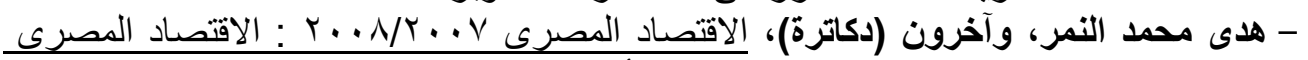

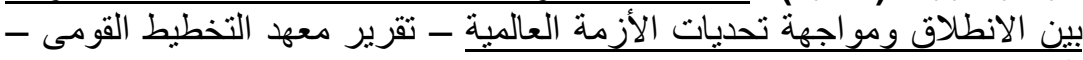

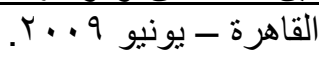

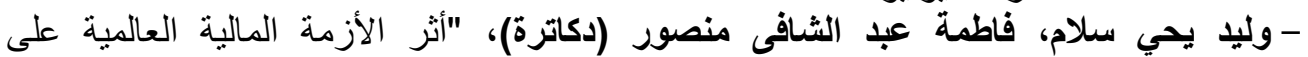

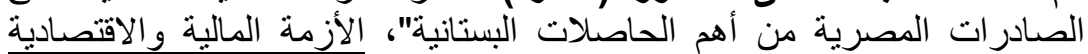

Fayoum J. Agric. Res. \& Dev., Vol.23, No.2, (B) July, 2009 
Ir.

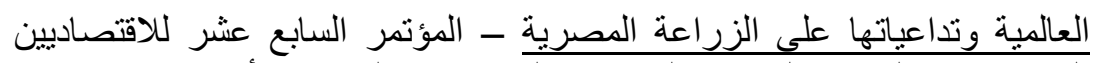

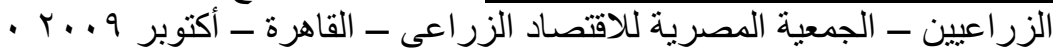

- European Commission - "EGYPT" COUNTRY STRATEGY PAPER 2007 - 2013 - January 2007.

- European Commission - Protocol to the Euro - Egyptian Association Agreement to take account of the accession of the Republic of Bulgaria and Romania to the European Union - Official Journal of the European Union, L312 - November 2007.

- European Commission - Protocol to the Euro - Egyptian Association Agreement to take account of the accession of the new ten members to the European Union - Official Journal of the European Union, L31

- February 2005.

- http://europa.eu.int

- http://www.un.org

- WAKASUGI Ryuhei (Dr) - International Trade during a Financial Crisis: WTO supervisory functions should be enhanced - Research Institute of Economy - January 2009.

\title{
THE IMPACT OF THE GLOBAL FINANCIAL AND ECONOMIC CRISIS ON THE EGYPTIAN AGRICULTURAL FOREIGN TRADE WITH THE EUROPEAN UNION
}

\author{
Rania Abd ElFatah Elshaer
}

Res. Agricultural Economics Res. Instit., Agric.Res.Center

\begin{abstract}
This research aims mainly to identify the implications of the global financial and economic crisis on the Egyptian foreign trade and agricultural foreign trade with the European Union in general and the major Egyptian agricultural exports to the EU in particular. The research indicates that the coverage rate of exports to imports has declined to about $46.2 \%$ in 2008 and so the deficit in Egyptian trade balance has increased by approximately $59.3 \%$ to almost 16.5 billion euros in the same year. It indicates also that the coverage rate of agricultural exports to agricultural imports has declined to approximately $26.5 \%$ in 2008 and so the deficit in Egyptian agricultural trade balance has increased by approximately $74.4 \%$ to about 4.32 billion euros in the same year. The research shows that the coverage rate of exports to imports with the EU has declined to about $63.6 \%$ in 2008 and so the deficit in Egyptian trade balance with the EU has increased by about $62.2 \%$ to about 4.64 billion euros in the same year. It shows also that the coverage rate of agricultural exports to agricultural imports with the EU has declined to about
\end{abstract}

Fayoum J. Agric. Res. \& Dev., Vol.23, No.2, (B) July, 2009 
$|r|$

$12 \%$ in 2008 and so the deficit in Egyptian agricultural trade balance with the EU has increased by approximately $93.9 \%$ to reach nearly 218 million euros in the same year, which reflects the negative impact of the global financial and economic crisis on the Egyptian trade balance and agricultural trade balance with world as well as with the EU. The research refers to increase the amounts and the values of the Egyptian exports of potatoes, grapes and oranges after the global financial and economic crisis, which reflects the absence of a negative effect of it on those exports. The research results show that there is no negative effect of the global financial and economic crisis on the comparative advantage of Egypt's export of potatoes, grapes and oranges to the EU where the value of the Export Specialization Index of each of those exports is more than the unit before the crisis and after it. They show also the increase of the market share of Egyptian exports of potatoes in the EU despite the global financial and economic crisis, which reduces the negative impact of the crisis on those exports which is the decrease of external demand for the European Union on potatoes reflected in the decrease of the amount of Egyptian potatoes exports to the European Union. The research shows increasing of the amounts and the values of the Egyptian exports of grapes and oranges to the EU, after the crisis, as well as growing market shares of those exports in the European Union during the periods having the Egyptian crop of those exports. The study recommends the need to adapt an agricultural economic policy include mechanisms and measures to address the economic crises when they occur in order to take prompt action to mitigate the effects and consequences of this crisis, as well as the need to reduce the geographic and commodity concentration for Egyptian agricultural exports which result in instability of the returns of those exports and its vulnerability to economic crises, and the need to strengthen the competitiveness of Egyptian agricultural exports and create new market opportunities in foreign markets, as well as the need to provide technical support to farmers to produce crops in conformity with the health and environmental standards to facilitate the penetration of global markets, in addition to that Egypt must negotiate with the EU on increasing the tariff quotas for the major Egyptian agricultural exports as well as modifying their export seasons to be consistent with their productive seasons in Egypt .

\begin{tabular}{|c|c|c|}
\hline \multicolumn{3}{|c|}{ 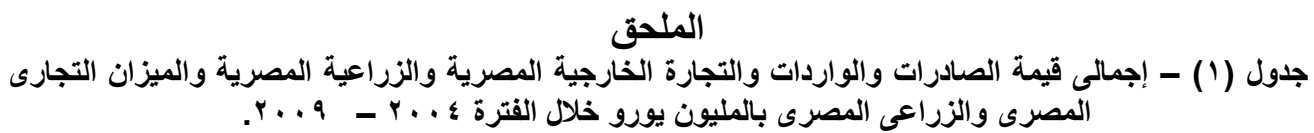 } \\
\hline \multirow[b]{2}{*}{$r \ldots 1$} & \multirow[b]{2}{*}{$r \cdots v-r \cdots \varepsilon$} & \multirow{2}{*}{ البيان السنوات } \\
\hline & & \\
\hline $1 \leqslant 104.91$. & $1 T K T 0.710$ & قيمة الصادر ات \\
\hline$r .701 .17 V$ & rrT99.097 & قيمة الواردات \\
\hline${ }^{*}(17 \leqslant 9 V .90 V)$ & ${ }^{*}(1.474 .911)$ & الميزان التجارى \\
\hline$\varepsilon \varepsilon \wedge .0 . V V V$ & 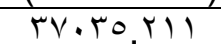 & إجمالى حجم التجارة الخارجية \\
\hline 1000.719 & 1477.719 & قيمة الصادر ات الزر اعية \\
\hline
\end{tabular}

Fayoum J. Agric. Res. \& Dev., Vol.23, No.2, (B) July, 2009 


\begin{tabular}{|c|c|c|}
\hline $0 \wedge V 7.0 V 1$ & $r V \leqslant \varepsilon .707$ & قيمة الواردات الزراعية \\
\hline$*(\varepsilon r Y \cdot . \wedge \wedge Y)$ & $*(Y \leq \vee \wedge . \cdot r V)$ & الميزان التجارى الزراعى \\
\hline VETY.YT. & 0.11. YVO & إجمالى حجم التجارة الخارجية الزر \\
\hline
\end{tabular}

المصدر: جمعت وحسبت من : بيانات موقع الأمم المتحدة ( www.un.org ) * الأرفام بين القوسين سالبة

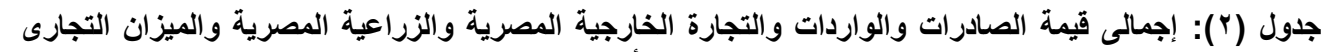

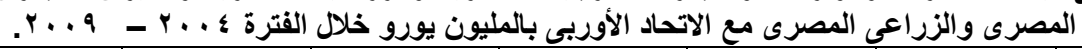

\begin{tabular}{|c|c|c|c|c|c|c|}
\hline 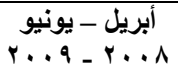 & 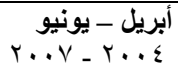 & 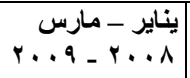 & 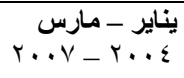 & $r \ldots \Lambda$ & $\begin{array}{c}-r \ldots \varepsilon \\
r \ldots v\end{array}$ & السنوات \\
\hline 19.7 .141 & 1071.1 .7 & $19.7 . V Y 1$ & $10 \ldots . Y 41$ & $\wedge \cdot \wedge 7.9 \vee 7$ & T.rA.IYT & قيمة الصادرات \\
\hline TYYT.VVI & rYAT.IA. & YA9Y.09\& & $1999 . Y V \varepsilon$ & ITVTI. $\leqslant 0 \leqslant$ & $1190 . \leqslant 7 \mathrm{~V}$ & قيمة الواردات \\
\hline${ }^{*}(1 M \mid \vee . T \varepsilon \cdot)$ & $\left.{ }^{*}(V) \leq, r V \varepsilon\right)$ & ${ }^{*}(৭ \wedge 0, \wedge \vee \top)$ & ${ }^{*}(\varepsilon 99 . \cdot 1 \%)$ & ${ }^{*}\left(\xi \neg \leqslant \leqslant \_\leqslant \vee \wedge\right)$ & ${ }^{*}(\Upsilon \wedge \odot \vee, \Gamma \varepsilon \varepsilon)$ & الميزان التجارى \\
\hline $01 r 9.9 . r$ & rᄉ01.914 & $\leqslant \vee 99.110$ & $r \leqslant 99.0 r \leqslant$ & $r \cdot \Lambda \backslash \Lambda . \leqslant r$. & $1 \leqslant 94 r .09$. & التجارة|الخارجية حجم \\
\hline r)9.97r & $\mathrm{N} \cdot .10 \mathrm{~V}$ & 417.119 & 194.100 & $719.00 Y$ & $009 . \vee 19$ & قيمة الصادرات \\
\hline$r r_{0} . T \leq V$ & $1 \leq V . \wedge 0$. & TrT. ET & IV9.1T人 & Arv.OYI & TVY.YIT & قيمة الواردات \\
\hline${ }^{*}(10.7 \wedge \varepsilon)$ & $r r . . V$ & ${ }^{*}(17.9 . \varepsilon)$ & Tr.TIV & $\left.{ }^{*}(Y) \vee .9 \vee \cdot\right)$ & ${ }^{*}((1 Y . \leq Y \leq)$ & الميزان التجارى الزراعى \\
\hline$\{00.71$. & $r 1 \Lambda . V \cdot V$ & $\leq \leqslant 9.1 \leq 1$ & $r v . \leqslant 9 T$ & $1 \leq 0 V . V T$ & Irrr...r & إجمالى حجية الزراعية التجارة \\
\hline
\end{tabular}

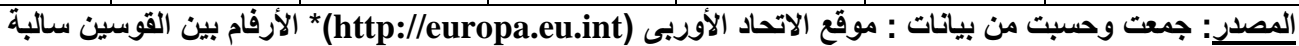

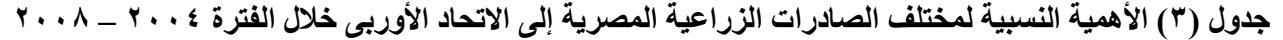

\begin{tabular}{|c|c|c|}
\hline الأهمية النسبية & متوسط قيمة الصادرات سنوياً & البيان \\
\hline $1 T_{.} \cdot 1$ & $7 \Lambda .7 \vee \wedge$ & البطاطس \\
\hline A.IT & $\leqslant\rceil . \leqslant \Psi \uparrow$ & العنب \\
\hline ^.11 & $\sum \tau$. «रY & البرتقال \\
\hline 7.11 & $r r_{.} \cdot v q$ & الفاصوليا الخضر اء \\
\hline 0.1. & 19.171 & الأرز \\
\hline$\varepsilon . \wedge \wedge$ & $Y V . \wedge \wedge V$ & القطن الخام \\
\hline r.rV & 19.YTr & البصل المجفف \\
\hline$r . \cdot A$ & IV.TYV & الفراولة \\
\hline$\sum 9 .+Y$ & $r \wedge . . r 01$ & منتجات أخرى \\
\hline $1 \ldots$ & OVI.V VI & الإجمالى \\
\hline
\end{tabular}

المصدر: جمعت وحسبت من بيانات: موقع الاتحاد الأوربى ( http:/europa.eu.int) الأرفام بين القوسين سالبة. جدول (؛ ): كمية ومتوسط السعر التصديرى لأهم الصادرات الزراعية المصرية إلى الاتحاد الأوربى خلال الفترة ؛ . .. -ـ

\begin{tabular}{|c|c|c|c|c|c|c|}
\hline \multicolumn{7}{|c|}{$.9 \cdots 9$} \\
\hline \multicolumn{2}{|c|}{ برتقال } & \multicolumn{2}{|c|}{ عنب } & \multicolumn{2}{|c|}{ بطاطس } & \\
\hline البالمليون يوريروى & الصادرات بالأف & الالملير التصديرى يورو & الصادرات بالأف & السبعليون يوريروى & الصادرات بالأف & \\
\hline$\varepsilon r \cdot .9 V \Gamma$ & 1.0 .119 & 1009.0Y. & TV.9VT & r90.711 & Yro.90. & $r \ldots V_{-} r_{\ldots} . . \varepsilon$ \\
\hline$\varepsilon 7 . .1 Y V$ & $1 . V .941$ & TEๆะ. Y. & rq. एवV & $r \leqslant 0.701$ & $T Y \cdot . \varepsilon q \lambda$ & $\ldots 1$ \\
\hline
\end{tabular}

Fayoum J. Agric. Res. \& Dev., Vol.23, No.2, (B) July, 2009 


\begin{tabular}{|c|c|c|c|c|c|c|}
\hline$\varepsilon \varepsilon 1, \Gamma \Gamma \xi$ & 01.91. & Tro.... & . & $r .0 . \Lambda . r$ & IVT.r $\Sigma \Gamma$ & 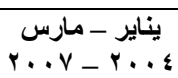 \\
\hline$\sum V O . \sum Y T$ & $V \cdot . r \varepsilon$ & $1770.1 Y \varepsilon$ & $\because \cdots \wedge$ & $r V \cdot . r 79$ & Irq.ra1 & 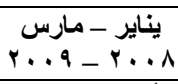 \\
\hline$\varepsilon r q . \vee V \varepsilon$ & $\varepsilon .0 .0$ & $10 \leqslant r . \Lambda . V$ & 17.00 & . & or. 9 9 & 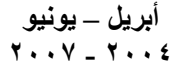 \\
\hline$\varepsilon \sum \Lambda .77 T$ & rq.rv. & IVAV.A99 & rT.ONA & $r \cdot \wedge .7 \ldots$ & $\vee 9 . \wedge .7$ & 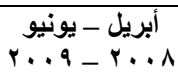 \\
\hline TAY. $\$ 90$ & $\cdot V 1 T$ & 101Y.Y70 & 11.49. & TAV.71. &. .015 & 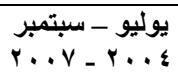 \\
\hline$r \leqslant 1 . \leqslant 99$ & E.oro & $15 \cdot 0.09 \varepsilon$ & $9 . \leqslant 9 \%$ & TVO.VOV & $\cdot .7 \cdots$ & يوليو - سبتمبر \\
\hline
\end{tabular}

المصدر: جمعت وحسبت من بيانات : موقع الاتحاد الأوربى (http://europa.eu.int ) * الأرفام بين القوسين سالبة .. أقل من ألف طن

جذول (0): كمية ومتوسط السعر الاستيرادى للاتحاد الأوربي من بعض السلع الزراعية خلال الفترة ؛ . . ب_a . . . . .

\begin{tabular}{|c|c|c|c|c|c|c|}
\hline \multicolumn{2}{|c|}{ برتقال } & \multicolumn{2}{|c|}{ عنب } & \multicolumn{2}{|c|}{ بطاطس } & \multirow{2}{*}{ "المنتج } \\
\hline بالمليون يورالتيرادى & الواردات بالالف & "السعرالاستيرادى & الواردات بالالف & بالمليون يورو الاستيرادى & الواردات بالالف & \\
\hline$O \cdots . \lambda r V$ & QYI.TAV & $1799.77 \varepsilon$ & 199.. 19 & $r 1 T .010$ & $0 \wedge \lambda . r \leqslant 9$ & $r \ldots V-r \ldots \varepsilon$ \\
\hline $0 Y \cdot .017$ & $1 \cdots 4 . \cdots r$ & TETr. $\cdot \Gamma \wedge$ & $901 . \cdot 1 Y$ & TVI.7Yq & 010.101 & $r \ldots \Lambda$ \\
\hline$\leqslant \vee 0.799$ & $1 \wedge . . \wedge 7$. & $1 \% \wedge 9 . \leqslant 50$ & Y৭४. \&YV & TrT.Y $\leq \leq \varepsilon$ & $r .0 .117$ & 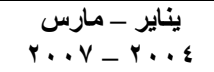 \\
\hline $01 \cdot . Y^{4}$ & 174.941 & $10 \leqslant 9.77 \leqslant$ & $r \cdot \Lambda .1 \times 4$ & $r \wedge 9.7 T$ & rTV.19V & 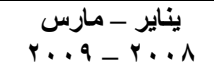 \\
\hline$\leq \pi), V Y \varepsilon$ & 17.947 & IYAT.rVq & ror..$\leqslant r$ & KIT.AAV & YO1.AMA & 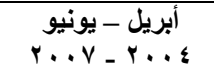 \\
\hline ort.l1T & $100.7 \leqslant 9$ & 1T4. ror & $r \cdots . \wedge \wedge 1$ & TIV.VYI & rYy.0Tr & 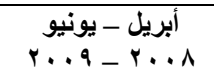 \\
\hline oro.r. 9 & 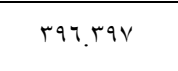 & $1.71 . V \times 1$ & ITH.ATY & KrY. $\leq \leq \varepsilon$ & $1 . .7 \leqslant V$ & 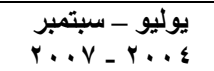 \\
\hline 0 - 9.109 & OTY.KYY & $1119 . \times 99$ & $1 \times 9 . \leqslant 00$ & TVY.AYA & $11 ., 4 \leq$ & 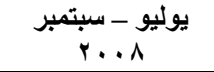 \\
\hline
\end{tabular}

المصدر: جمعت وحسبت من بيانات : موقع الاتحاد الأوربى (http:/europa.eu.int) الأرفام بين القوسين سالبة

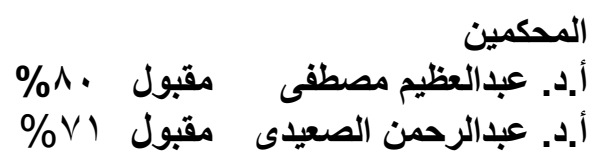

Fayoum J. Agric. Res. \& Dev., Vol.23, No.2, (B) July, 2009 